\title{
Orion Flight Performance Design Trades
}

\author{
Mark C. Jackson ${ }^{1}$ \\ Charles Stark Draper Laboratory, Houston, TX, 77062 \\ and \\ Timothy Straube ${ }^{2}$ \\ NASA Johnson Space Center, Houston, TX, 77058
}

\begin{abstract}
A significant portion of the Orion pre-PDR design effort has focused on balancing mass with performance. High level performance metrics include abort success rates, lunar surface coverage, landing accuracy and touchdown loads. These metrics may be converted to parameters that affect mass, such as ballast for stabilizing the abort vehicle, propellant to achieve increased lunar coverage or extended missions, or ballast to increase the lift-to-drag ratio to improve entry and landing performance. The Orion Flight Dynamics team was tasked to perform analyses to evaluate many of these trades. These analyses not only provide insight into the physics of each particular trade but, in aggregate, they illustrate the processes used by Orion to balance performance and mass margins, and thereby make design decisions. Lessons learned can be gleaned from a review of these studies which will be useful to other spacecraft system designers. These lessons fall into several categories, including: appropriate application of Monte Carlo analysis in design trades, managing margin in a highly mass-constrained environment, and the use of requirements to balance margin between subsystems and components. This paper provides a review of some of the trades and analyses conducted by the Flight Dynamics team, as well as systems engineering lessons learned.
\end{abstract}

\section{Introduction}

$\mathrm{T}$ The Orion Crew Exploration Vehicle (CEV) consists of three major components (Figure 1): a Crew Module (CM), Service Module (SM) and a Launch Abort System (LAS). The requirement for Orion to carry four crew members to and from the Moon has resulted in a larger, heavier CM than the Apollo design. The capsule diameter is $16.5 \mathrm{ft}$, nearly $4 \mathrm{ft}$ larger than the Apollo capsule with 2.5 times the internal volume. This larger capsule requires a larger, more powerful LAS to achieve required separation during launch aborts. The SM, on the other hand is smaller than its Apollo counterpart, since the task of inserting the vehicle into lunar orbit falls to the lunar lander propulsion system, rather than the SM engine, as in Apollo. Even with the smaller service module though, mass has proven to be a critical design parameter for Orion. The mass of the Orion CM affects abort system performance, landing loads, parachute sizing, launch vehicle performance and translunar injection (TLI) performance. The mass of the SM drives the range

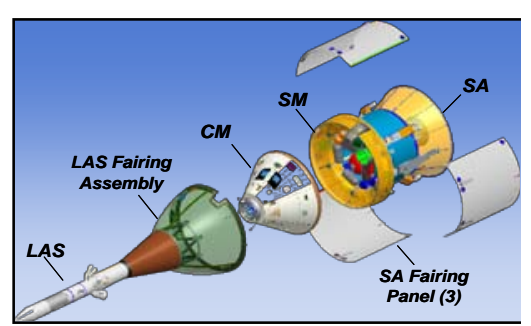

Figure 1. Orion Components of lunar surface sites that are achievable at a given Earth-Moon geometry as well as launch and TLI performance.

A significant portion of the Orion pre-PDR design effort has focused on balancing mass with performance. High level performance metrics include abort success rates, lunar surface coverage, mission duration limits, landing opportunity rates and landing accuracy. These metrics may be converted to parameters that affect mass, such as ballast for stabilizing the abort vehicle, propellant to achieve increased lunar coverage or extended missions, or ballast to increase the lift-to-drag ratio to improve entry and landing performance. The Orion Flight Dynamics team was tasked to perform analyses to evaluate many of these trades. These analyses not only provide insight into the

\footnotetext{
${ }^{1}$ Insert Job Title, Department Name, Address/Mail Stop, and AIAA Member Grade for first author.

${ }^{2}$ Insert Job Title, Department Name, Address/Mail Stop, and AIAA Member Grade for second author.
} 
physics of each particular trade but, in aggregate, they illustrate the processes used by Orion to balance performance and mass margins, and thereby make design decisions. Lessons learned can be gleaned from a review of these studies which will be useful to other spacecraft system designers. These lessons fall into several categories, including: appropriate application of Monte Carlo analysis in design trades, managing margin in a highly massconstrained environment, and the use of requirements to balance margin between subsystems. This paper provides a review of some of the trades conducted by the Flight Dynamics team.

The following sections describe selected trade studies whose results quantified the sensitivities of vehicle performance to mass. Trade study descriptions are divided into ascent abort, orbit, and entry sections. The ascent abort section covers a trade on the stability of the abort vehicle versus ballast mass. Orbit trades selected are "fail safe" Moon-to-Earth transfer maneuvers and Lunar coverage studies. Entry studies include entry performance versus ballast mass, and active roll control performance under chutes to orient the vehicle at touchdown. Each trade study contains a summary of the results in terms of performance margin and sensitivity of performance to mass.

After the trade study discussions we use the margin and sensitivity data to provide a notional example of the margin balancing that is performed at the Orion project level. The example highlights many of the issues that must be dealt with by Orion systems engineers and provides a conceptual framework for using the study information

The final sections present lessons learned from the conduct of the studies and from the application of their results in vehicle-level integration. These include lessons on simulation and Monte Carlo analysis - from avoiding traps during the analysis to interpreting the results correctly.

\section{Definition of Margin and Sensitivity}

To aid in better defining margin and sensitivity for the rest of this paper, it is useful to define a relative performance metric. This metric is the performance requirement, divided by the measured performance. Using landing accuracy as an example, if a study shows that 3 times the standard deviation $(3 \sigma)$ of landing accuracy for a particular set of parameters is 4 nautical miles, and the required landing accuracy $(3 \sigma)$ is $5 \mathrm{~nm}$, then the relative performance metric is 1.2 - so this relative metric equals one when the performance equals the requirement. Margin then, is the relative performance metric minus one. In the landing example, margin is 0.2 or $20 \%$.

Sensitivity is normally thought of as the partial derivative of the performance metric to a cost variable. The cost variable used here is mass, but other cost metrics are possible, including monetary cost. In the landing example, if adding $100 \mathrm{lb}$ of ballast to the $\mathrm{CM}$ increases landing accuracy by 0.2 nautical miles, then the sensitivity of accuracy to mass is $0.2 / 100$ or $0.002 \mathrm{~nm} / \mathrm{lb}$. This type of sensitivity however does not allow the systems engineer to trade different types of performance. Which is worth more, for example, landing accuracy or lunar surface coverage?

The measure of "importance" of a particular performance metric is the requirement. So for comparison to other performance metrics, we want the sensitivity of margin with respect to the required performance. In the landing example, if the additional mass improves landing accuracy to $3.8 \mathrm{~nm}$, then margin is increased to $22 \%$ and the sensitivity of the margin to mass (in percent) is 100 times the performance sensitivity divided by the requirement, or 0.04. We will use this type of sensitivity (margin to mass) in our examples derived from the trade studies below.

\section{Ascent and Abort Trade Study Example}

During ascent, Orion may abort from a failing, or under-performing launch vehicle in two ways. During first stage flight, a Launch Abort System (LAS) is provided to remove the Crew Module (CM) capsule from a booster experiencing catastrophic failure. Shortly after first stage burnout, the LAS is jettisoned and the responsibility for separation from the launch vehicle falls to the Service Module (SM). Second stage booster failures are generally considered more benign that first stage failures. This section describes one of many trades conducted to help design the LAS.

\section{A. Stability vs Ballast}

The Orion Launch Abort System (LAS) consists of three rocket motors housed in a tower-fairing assembly (Figure 2). The abort motor provides the thrust to remove the Launch Abort Vehicle (LAV) - the combined LAS and capsule - from the malfunctioning booster. The jettision motor detaches the LAS from the capsule after it has been re-oriented to a heat shield forward attitude during the abort. The attitude control motor (ACM) is a solid rocket engine whose thrust is directed through a ring of eight "pintle" valves. The net thrust vector is controlled by opening or closing valves in the ring. The role of the ACM is to stabilize and control the LAV during the rocket motor burn, and to re-orient the LAV to the heat-shield forward attitude for LAS jettison and subsequent chute deploy.The Orion abort system is required to prove a $95 \%$ success rate averaged over the ascent time period. A further design objective is to eliminate specific periods during the ascent with low success probabilities. 


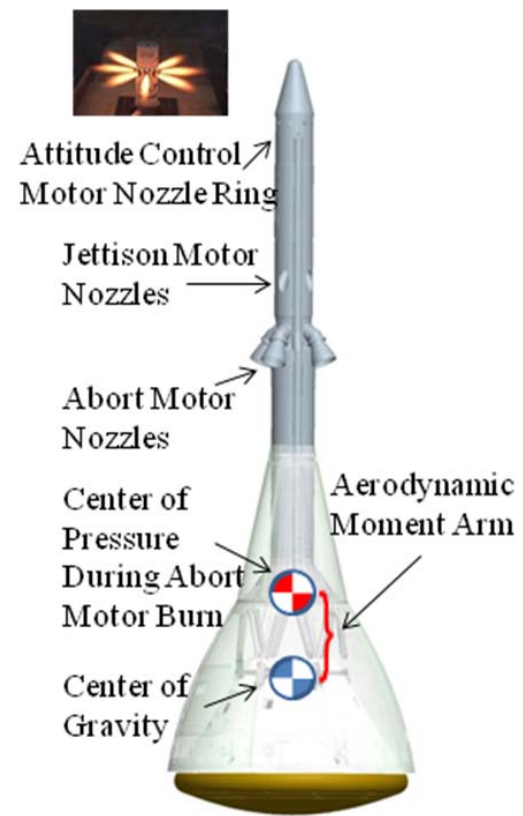

Figure 2. Launch Abort Vehicle

One of the challenges of controlling the LAV is that the abort motor thrust plumes move the aerodynamic center of pressure ahead of the center of gravity making the LAV dynamically unstable during the abort burn (Figure 3). In addition to the normal challenges associated with un-stable vehicles, the forward center of pressure increases the aerodynamic moment arm for pitch and yaw. This means that large aerodynamic torques may occur when the vehicle is displaced from the trim angle of attack.

The degree to which the abort motor plumes affect the center of pressure is difficult to determine precisely. The resulting uncertainty in pitch moment torque means that under certain conditions the aerodynamic pitch moment may exceed the available torque from the ACM control motor. Should this occur the vehicle will tumble out of control.

The combination of

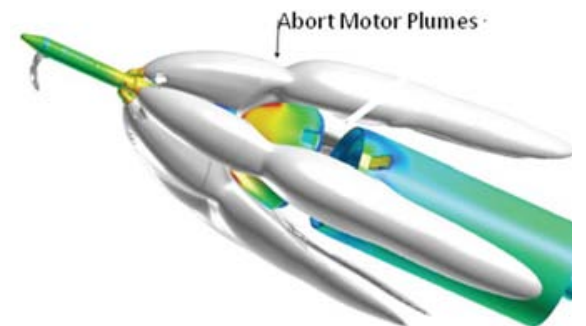

Figure 3. Abort Motor Plumes

large aerodynamic uncertainties together with increases in the CM mass allocation, resulted in the vehicle tumbling during a significant number of simulated abort cases - especially when aborts occurred around Mach 1. To correct these tumbles, weight or ballast may be added in the top portion of the LAS tower. This moves the center of gravity forward, reducing the aerodynamic moment arm of Figure 2.

To determine the effect of ballast on abort success, a Monte Carlo simulation study was conducted with varying amounts of ballast. Figure 4 summarizes the tumbling failure rates for several quantities of ballast. Aborts were initiated at altitudes between zero (the launch pad) and around $65000 \mathrm{ft}$ - the point at which the LAS is jettisoned after first stage separation. Parameters varied in the Monte Carlo included aerodynamic parameters, mass properties and rocket motor parameters.

The figure shows that with no ballast (blue curve) there are altitude regions in the ascent profile for which the probability of abort failure due to tumbling are relatively high - more than $35 \%$ at the peak and greater than $5 \%$ for a large section of the ascent. The improvements in success rate are clear from the reduced tumbling cases associated with even the relatively "small" $660 \mathrm{lb}$ nose-cone ballast.

The results of this study provided Orion management and systems decision makers data on the success rate as a function of ballast mass. As we shall see, the mass data may be used as a common "currency" to trade first stage abort success performance against other spacecraft attributes which enhace crew safety, improve capability or increase mission success rates. When considering the ballast mass numbers against other sources of mass on the Orion vehicle, bear in mind that, since the LAS is jettisoned after first stage, mass on the LAS is not as critical to ascent performance as other vehicle mass which is fully lifted into orbit. A rule of thumb calculation for comparing LAS mass to CM or SM mass is that one pound of LAS mass affects ascent performance about $1 / 8$ as much as one pound of CM or SM mass.

In order to balance margins across the vehicle design, we must quantify the margin on vehicle-level requirements. That presents some difficulty with the success rate requirements that apply to the LAS. For example, consider the LAV requirement of $95 \%$ success. Even if the

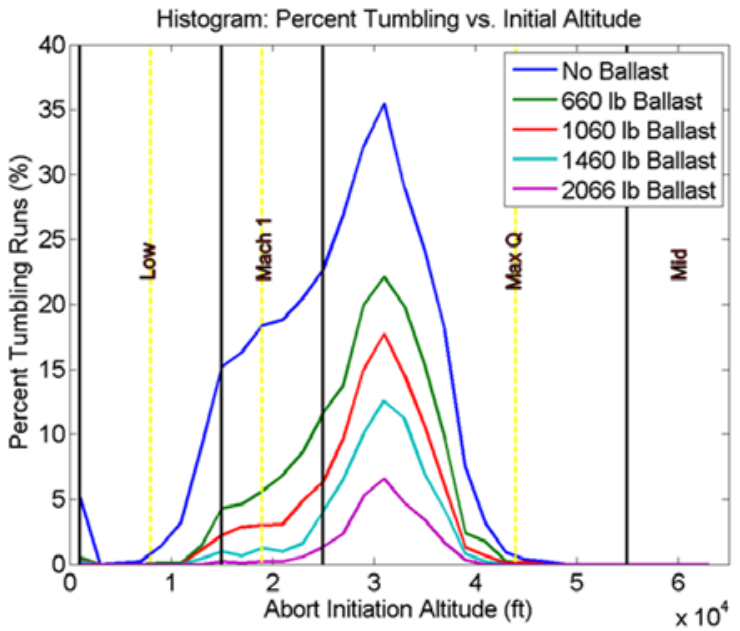

Figure 4. Effect of Ballast on Tumbling 
vehicle is successful $100 \%$ of the time, the margin on this requirement is only $5 \%$ so standard margins of $30 \%$ at PDR and $10 \%$ at CDR are not acheiveable. Further, this limit on maximum margin precludes margin balancing since no amount of ballast assures $100 \%$ success. For these reasons, we have taken the approach of redefining margin based on a fictitious "underlying performance parameter." Failure is presumed to occur when that parameter exceeds a limit. The details of this process are provided in the appendix since, although they are interesting, they are not germain to the overall process of trading LAS abort performance.

Although the 95\% time-averaged success requirement does not preclude high failure rates at particular points along the profile, the flight dynamics team has the design goal of limiting failure rates along the profile. As of this writing, the vehicle level trades are not complete to determine how much, if any ballast will be allocated. However, for the purposes of the margin balancing discussion below, we will assume that the amount of ballast used should be sufficient to keep success rates above $80 \%$ at all points along the profile. This assumption also simplifies the determination of margin and sensitivity, since we now need only consider the data at the peak failure rate - a little more than 30,000 ft in Figure 4.

Table 1 summarizes the LAV representative performance requirements, margins and sensitivities gleaned from this and similar Monte Carlo studies. Note that with no ballast the LAV has negative margin on the success requirement.

Table 1. Margin and Sensitivity for LAV Ballast Trade

\begin{tabular}{|l|l|c|c|}
\hline Trade & Requirement/ Design Objective & Margin & Sensitivity (\%/lb) \\
\hline Success vs. Ballast Mass & $80 \%$ Success at any altitude & $-19 \%$ & 0.23 \\
\hline
\end{tabular}

Of course, other parameters affect performance as well as ballast. Expected performance may be improved by reducing aerodynamic uncertainties through flight or wind-tunnel tests, by improving the control design, or by any number of other techniques. But as Orion approaches CDR the requirements, control design and major environmental models will become more stable, and final decisions will be made to allocate mass according to the remaining vehicle-level "knobs" such as ballast and propellant.

\section{Orbit Trades}

This section presents two trade studies conducted to relate Orion performance to SM propellant mass. The first study looked at the techniques used in targeting and executing main engine burns at the moon. This study was used to determine the assumptions that should be used in generating propellant budgets for lunar missions. The second study determined available lunar surface coverage as a function of SM propellant.

\section{A. Fail Safe Trans Earth Injection}

During the lunar mission, after the Altair lander has returned the crew to Orion in lunar orbit, the Altair ascent stage is jettisioned, and the Orion SM performs the series of burns at the Moon to initiate the orbital transfer back to Earth. A typical three burn, trans-Earth injection (TEI) series is depicted in Figure 5. TEI-1 transfers Orion from a near circular low lunar orbit (LLO) to a highly elliptical orbit (yellow arc). Near the apoapse of this orbit, TEI-2 is executed to place the vehicle in a lunar orbit plane that corresponds to the plane of the desired Earth-relative velocity for the return trip. TEI-3 is then executed to accelerate away from the Moon and enter an Earth-relative orbit with

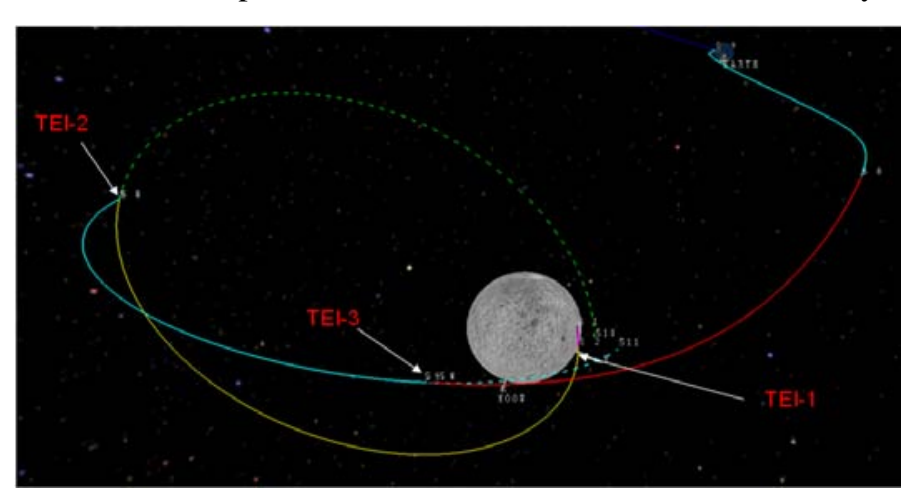

Figure 5. Trans-Earth Injection (TEI) Sequence the desired periapse for Entry. On the return trip, trajectory correction maneuvers (TCMs) are executed to correct dispersions from the ideal TEI sequence and to precisely target the entry interface conditions.

During the TEI sequence, two types of failures can cause the spacecraft to impact the Moon: 1) failure to execute a burn - which can cause impact if the current orbit has a perigee below the lunar surface or, 2) an engine failure during a burn - which can cause impact if the resulting orbit has a perigee below the surface.

4

American Institute of Aeronautics and Astronautics 
The first of these failures - failure to execute - results in impact if the previous burn left the spacecraft in an orbit with a low perigee. This is a function of how the previous burn is targeted and can occur if, for example, the

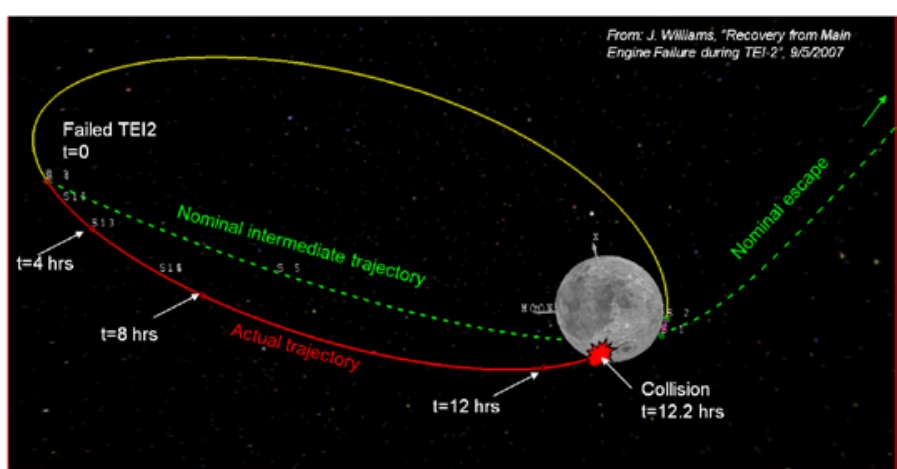

Figure 6. Impact Resulting from Failed Burn Execution burn had a radial component. Experience has shown that a fuel optimal burn sequence can result in orbits with unsafe periapse. Figure 6 shows an example in which TEI-1 is executed to target the optimal TEI-2 position resulting in an orbit that impacts the lunar surface if TEI-2 is not executed. As the figure shows, from the time that TEI-2 fails to execute ( $\mathrm{t}=0$ ) to lunar surface impact is 12.2 hours. For this particular failure then, some time remains to correct the problem, prevent lunar impact, and re-target the return.

This situation may be contrasted with that of Figure 7. In this example, TEI-2 is executed normaly, but the optimal solution leaves Orion in a lunar impact orbit. This solution relies on TEI-3 executing properly to prevent collision with the Moon, and - as the figure shows - only leaves about 12 minutes to correct a problem once the failure is discovered at TEI-3 execution.

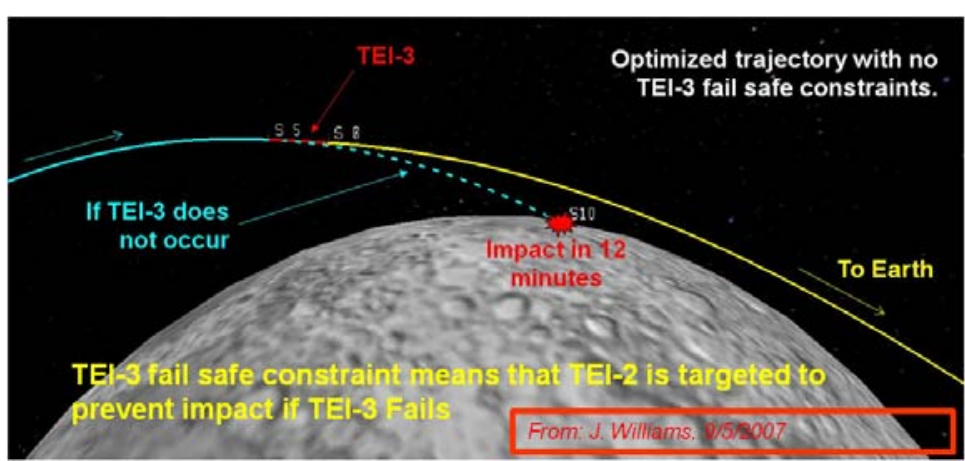

Figure 7. Impact Resulting from Failed TEI-3 Execution

The collisions depicted in Figures 6 and 7 may be prevented by adopting a policy constraining the targeting of all burns to result in periapse above a prescribed altitude. This policy is referred to here as "fail safe targetting."

The second type of failure considered is an engine failure during the burn. Figure 8 is a conceptual diagram of the velocity change executed at TEI-2. In this example, the velocity direction is changed by $90^{\circ}$ at or near apoapse, resulting in an orbit whose plane is consistent with the orbit plane needed for Earth return. The red traces in the figure represent two candidate burn execution strategies. On the left the "chord burn" is executed in the direction to most efficiently and directly change the velocity from the intial orbital velocity to the final desired. However, if the burn should stop for any reason along the red trace, the resulting velocity magnitude is less than the initial and final velocities. This smaller velocity magnitude may result in an orbit with lower periapse which impacts the moon.

In contrast, the fail safe burn is executed so that the resulting velocity magnitude always results in a safe orbit - at the cost of the extra propellant corresponding to the longer red arc.

The fail safe TEI trade study

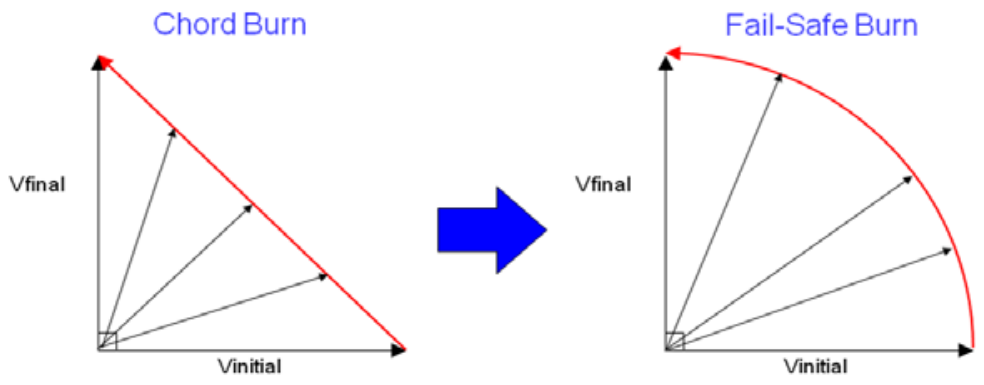

Figure 8. Fail Safe Burn Geometry quantified the propellant mass costs for both fail safe targeting and fail safe execution. Propellant numbers are omitted here since spacecraft performance may be sensitive, but the study recommandations may be understoon from the above discussion. They were:

- TEI-1 should be targeted to prevent low periapsis since the fuel penalty was always very small

- TEI-2 execution is not required to be fail safe since many hours are available to correct the resulting orbit, and since auxiliary thrusters are available to back up the Orion main engine (OME)

- TEI-2 is targeted to guarantee a safe periapsis since little time remains after a TEI-3 failure 
This trade study did not quantify margin with respect to a requirement. Rather, it resulted in a policy for use in other design trades, such as the following lunar suface coverage trade study.

\section{B. Lunar Geographic and Temporal Coverage}

The Orion concept of operations allows for two types of lunar missions. Lunar outpost missions are intended to service a lunar base at the Moon's south pole. During outpost missions, Orion loiters unmanned in LLO for up to six months. Lunar sortie missions are intended to allow exploration of any portion of the lunar surface and are much shorter than lunar outpost missions - lasting around 18 days under nominal conditions with a week on the lunar surface.

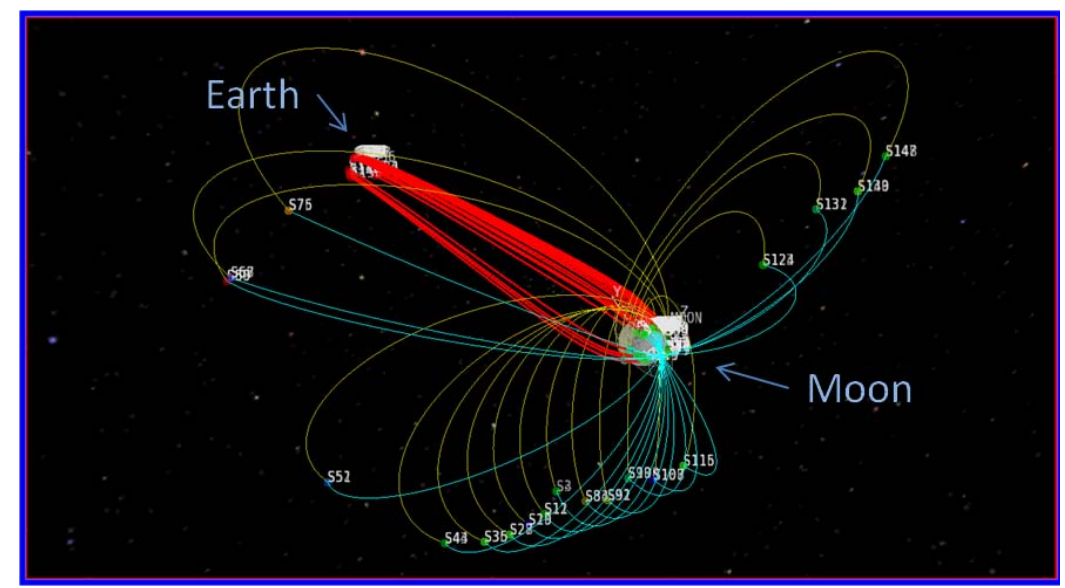

Figure 9. Return Trajectories from Assorted Landing Site

In order to size Orion propellant loads and tank capacities, it is useful to relate the amount of the lunar surface that can be reached to propellant mass. To this end, it is useful to define two types of lunar coverage: geographic coverage, and temporal coverage. Geographic coverage is the amount of the Moon's surface that may be reached at a given launch epoch while temporal coverage is the percentage of time (over the 19 year Metonic cycle) that a particular site can be reached. Given prescribed launch vehicle and lander capacities, the reachable portions of the Moon are determined by Orion's capacity to change it's lunar orbit plane to pass over the landing site, and then reach an appropriate entry interface condition via the three burn TEI sequence described above. This capacity depends on the total inertial velocity change, $\Delta \mathrm{v}$, that Orion can produce, which in turn depends on the specific impulse $\left(\mathrm{I}_{\mathrm{sp}}\right)$ of the engines, and the amount of onboard propellant.

Figure 9 shows the TEI sequences and return trajectories for several landing sites at constant latitude and varying longitude around the Moon. From the figure, it is clear that the TEI burn sequence associated with a particular site has a large effect on the total $\Delta \mathrm{v}$ required to return from that site. So if the propellant load is smaller than that required for the worst of these trajectories, then some percentage of these sites will not be available for exploration at a given epoch.

Figure 10 summarizes the results of a study conducted to determine site availability as a function of propellant load. The study developed mission designs (sequences of burns) for a grid of landing sites around the Moon across a set of departure epochs that spanned the Metonic cycle which governs Earth-Moon geometry. The study consisted of nearly 1 billion site-epoch combinations.

The horizontal axis of the figure is the onboard propellant in $\mathrm{Kg}$ and the vertical axis shows the percentage of the lunar surface that may be reached given a particular propellant load and a desired

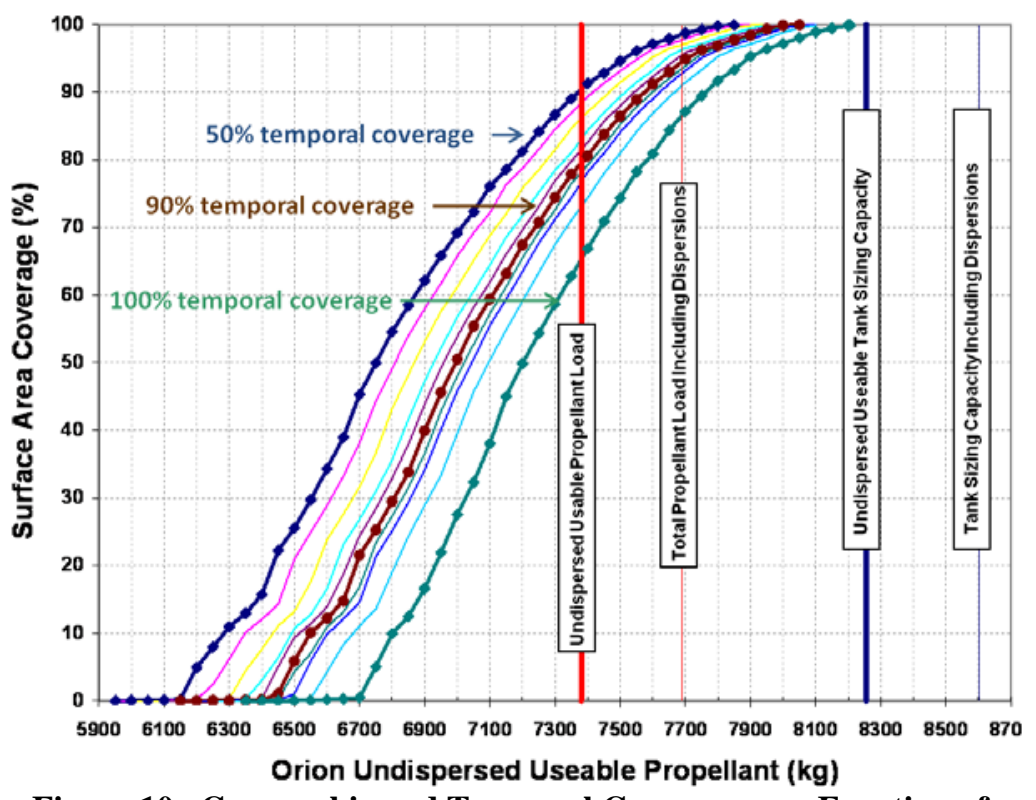

Figure 10. Geographic and Temporal Coverage as a Function of Propellant Load 
amount of temporal coverage. The curves on the graph are the temporal coverage available at a given combination of propellant load and surface coverage.

To read the graph, start with a desired propellant load - say the usable propellant load indicated by the thick red vertical line. Follow the line up to a desired temporal coverage curve - say $90 \%$ temporal coverage. The intersection of the red propellant line with the $90 \%$ curve occurs at about $80 \%$ surface coverage. This means that with around $7400 \mathrm{Kg}$ of useable propellant, Orion can reach $80 \%$ of the Moon's surface $90 \%$ of the time. For the same propellant load, Orion can reach about $65 \%$ of the surface $100 \%$ of the time.

The solid red boundary in the figure is the total propellant required to ideally actuate the mission $\Delta \mathrm{v}$ assuming no targeting, navigation or control errors. Propellant must be added to the ideal value to account for these errors, or dispersions, in order to fly the real mission. This additional propellant is indicated by the thin red boundary in the figure. Margin may also be added to the total propellant load to account for uncertainties or contingencies. The thin red boundary may be thought of as the "design fuel load" since it represents the amount of fuel that is accounted for when trading propellant mass against other forms of mass (strucuture, ballast, heat shield, crew supplies, etc). The design fuel load may be viewed as a requirement which may be adjusted by systems engineers to balance margin.

For our purposes, we will consider the $100 \%$ temporal coverage of $65 \%$ of the surface to be the propellant defining requirement. Recall that the corresponding propellant also provides considerably more surface coverage during more favorable ephocs. Table 2 contains notional values (actual values may be sensitive) of the margin that the design propellant load contains. The sensitivity of surface coverage margin to propellant mass is also provided.

Table 2. Margin and Sensitivity for Lunar Surface Coverage Trade

\begin{tabular}{|l|l|c|c|}
\hline Trade & Requirement/ Design Objective & Margin & Sensitivity (\%/lb) \\
\hline $\begin{array}{l}\text { Surface Coverage vs. Propellant } \\
\text { Mass }\end{array}$ & $\begin{array}{l}65 \% \text { of lunar surface acheiveable on } \\
100 \% \text { of launch epochs }\end{array}$ & $20 \%$ & 0.12 \\
\hline
\end{tabular}

Finally, we note that the thin blue boundary in the Figure 10 represents the total propellant available in the SM tanks. Tank capacity exceeds the design fuel load to provide operational flexibility and future performance growth should other mass opportunities manifest themselves. Note that the dispersions must also be subtracted from the tank sizing capacity to get the "useable" fuel that may be ideally converted to $\Delta \mathrm{v}$.

\section{Entry Trades}

Two trades from the entry flight phase further illustrate the methods used to relate performance to mass. The first relates the capsule lift-to-drag ratio to ballast mass, and the second trades touchdown landing performance with structural mass.

\section{A. Entry Performance vs Ballast Mass}

After entering the atmosphere, the Orion CM seeks a "trim" attitude that balances aerodynamic torques. Figure 11 diagrams some the parameters which determine the pitch trim angle. In the figure, $\alpha$, or angle of attack is the

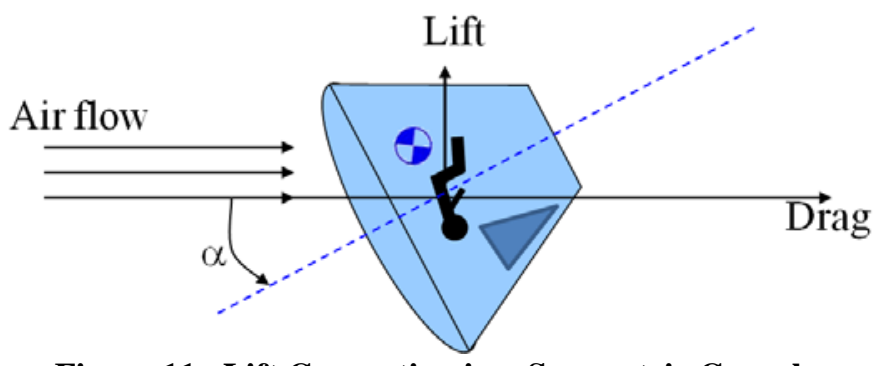

Figure 11. Lift Generation in a Symmetric Capsule angle between the relative air flow and the longitudinal axis of the vehicle (blue dashed line). At small angles of attack, the aerodynamic center of pressure on the CM is close to the center of the heat shield. When the center of gravity is offset from the longitudinal axis, a torque is generated about the CG that equals the aerodynamic force times the moment arm from the longitudinal axis to the CG. This torque causes the capsule to rotate in pitch until this torque is balanced by the restoring torques that occur from the symmetrical cone's tilt. At steady state then, the magnitude of the trim angle of attack depends on the CG offset from the longitudinal axis. This value tends to be fairly constant through hypersonic and supersonic flight. 
The tilt of the symmetrical cone-shaped capsule at trim creates lift in the direction of the CG offset. As depicted in the figure, the CG offset is constructed to create lift in the general direction of the crew's feet. This upside down posture, allows the crew to view the horizon out of the capsule windows.

At speeds above the transonic region, the capsule is stable at trim angle of attack, and aerodynamic torques resist perturbations in angle of attack away from trim. For this reason, pitch and yaw are not directly controlled once in the atmosphere, instead the reaction control thrusters are used to damp pitch and yaw rates to reduce oscillation about trim. In fact, once in the atmosphere, pitch and yaw control torques are insufficient to overcome aerodynamic torques, so controlling to an attitude far from trim is not possible.

The bank angle of the capsule on the other hand, is controlled - primarily by the roll RCS thrusters. Control of the bank angle determines the orientation of the lift vector about the relative airflow. The figure depicts zero bank or "lift up." By modulating bank, the direction of lift may be controlled to determine the capsule sink rate. Higher sink rates cause the capsule to encounter denser air more quickly, thereby increasing drag and shortening the downrange flown. In general then, a constant zero bank profile minimizes sink rate and maximizes range, while a constant $180^{\circ}$ bank, or "lift down" profile minimizes range. Guidance may command bank angles between zero and $180^{\circ}$ to achieve the range of the landing site. The resulting heading changes are small, and are compensated for by periodically reversing the bank direction to keep the heading azimuth within preset limits of the landing site bearing.

At zero angle of attack, lift is zero and all aerodynamic force is opposite the air flow in the direction of drag. So the amount of lift generated depends on the CG offset. A particular offset determines the trim angle of attack, which in turn determines the ratio of lift to drag, or $\mathrm{L} / \mathrm{D}$ ( " $\mathrm{L}$ over $\mathrm{D}$ "). $\mathrm{L} / \mathrm{D}$ is approximately constant from entry enterface to near the drogue deploy point. Larger values of L/D expand the available "footprint" of possible landing sites from a given entry interface condition.

Since $\mathrm{L} / \mathrm{D}$ is determined by the CG offset, the CM designers achieve as much offset as possible by packaging heavy spacecraft components in the desired offset direction. For Orion, this has not resulted in CG offsets which meet L/D requirements, so additional ballast must be added on the edge of the capsule cone to create more offset. A design trade must then be performed between the ballast mass, and the

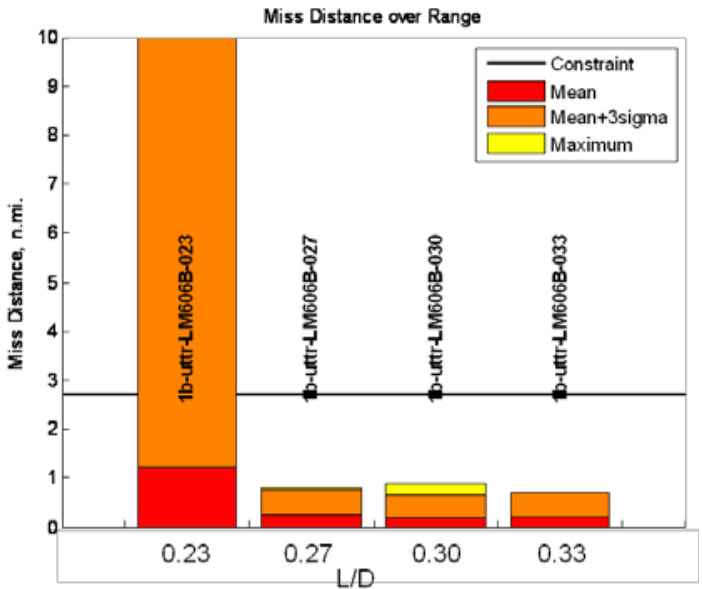

Figure 12. Sensitivity of Miss Distance to L/D performance associated with increased L/D.

To determine the minimum L/D, a series of Monte Carlo analyses were conducted at various entry geometries for both lunar and low Earth orbit (LEO) missions. Figure 12 is an example of the results for lunar return with a constant targeted flight path angle. Note that miss distance is well within the requirement for $\mathrm{L} / \mathrm{D}$ values above 0.23 and the sensitivity of miss remains small with decreasing L/D until suddenly increasing. This "cliff" behavior is due to the fact that the spacecraft can no longer fly the required range to the site with insufficient lift.

From these data, the requirement for L/D may be stated as: "The mean lift-to-drag ratio minus three times the standard deviation of the lift-to-drag ratio shall be greater than or equal to 0.23 .”

Table 3 contains notional values of the L/D margin that the design ballast mass provides. The sensitivity of L/D to ballast mass is a fairly straightforward calculation and is detailed in the appendix?

Table 3. Margin and Sensitivity for $L / D$ versus Ballast Trade

\begin{tabular}{|l|l|c|c|}
\hline Trade & Requirement/ Design Objective & Margin & Sensitivity (\%/lb) \\
\hline L/D vs Ballast Mass & $\begin{array}{l}\text { Mean L/D }-3 * \sigma \text { of L/D >= } 0.23 \\
\text { (lunar) }\end{array}$ & $15 \%$ & 0.05 \\
\hline
\end{tabular}

It should be noted that a maximum $\mathrm{L} / \mathrm{D}$ requirement is also enforced, since high $\mathrm{L} / \mathrm{D}$ values (and their associated trim angles of attack) can cause violations of thermal constraints. However, the minimum L/D value minimizes ballast mass, so it is of most interest for this discussion. More information about L/D constraints and their associated statistical limitations on CG placement, see reference XXX. 


\section{B. Roll Orientation Trades at Touchdown}

The Orion crew module is required to have enough habitable volume to carry four astronauts to the Moon with associated life support systems and expendables. The resultant capsule diameter is $16.5 \mathrm{ft}$, nearly $4 \mathrm{ft}$ larger than the Apollo capsule with 2.5 times the internal volume. The larger capsule diameter presents structural design problems, especially during water landings in high sea states. Landing impact attenuation systems designed to protect the crew from, one-chute-out

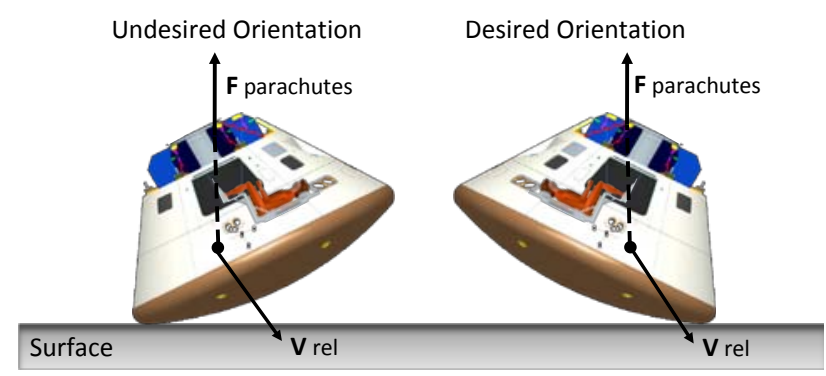

Figure 13. Desired Landing Orientation touchdown loads require increased mass and volume to handle worst-case combinations of sink rate, horizontal velocity, and wave impact geometry.

In order to mitigate the risk of structural failure or crew load exceedance the orientation of the capsule is controlled at touchdown to provide the best direction of impact for the attenuation system and to minimize the probability of impacting a wave in a "belly flop" orientation. Figure 13 illustrates the desired orientation with respect to the surface-relative velocity at impact $\left(\mathrm{V}_{\text {rel }}\right)$. Since the horizontal component of the relative velocity is due primarily to wind, changes in wind direction in the final $1500 \mathrm{ft}$ or so prior to touchdown result in changes in relative velocity which must be tracked by the on-board control system.

The ability to respond to changes in horizontal velocity is a function of velocity measurement accuracy and control response time. Accurate measurement of small, wind-driven horizontal velocities requires GPS. Even with GPS accuracy, however, the accuracy depends on velocity magnitude, which at steady state equals wind speed. So high winds, resulting in large horizontal velocities are easily measured, while low winds make velocity direction determination difficult. Fortunately, roll orientation at low relative speed is not critical for crew loading or structural deformation.

Control response is also a factor in achieving the desired orientation. When wind direction changes within the last few feet of the descent, the control system fires roll thrusters to turn the vehicle toward the new direction. The change in orientation may cause the main chute risers to twist, creating a back-torque disturbance with which the controller must contend. Further the controller is required to have a high success rate with the loss of one of the two redundant strings of CM thrusters, so control authority may be reduced in the worst case. Fortunately, in high-wind situations, wind direction variability is reduced and the wind direction is more likely to persist.

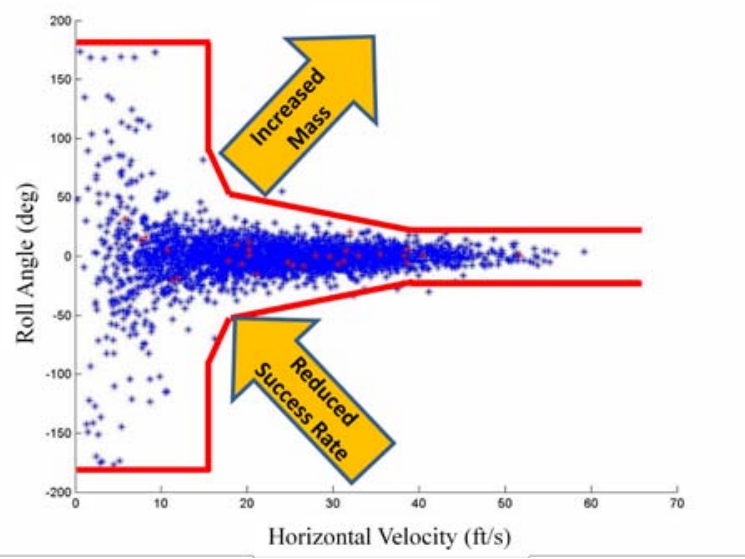

Figure 14. Roll Heading Error versus Horizontal Velocity

Figure 14 is a plot of the touchdown roll error against horizontal velocity magnitude for a Monte Carlo study which modeled wind variations, chute dynamic parameter variations, and navigation errors. The red boundary is the requirement for roll control accuracy, and reflects the maximum allowable value of three times the standard deviation of the error. Note that when the horizontal velocity is less than $10 \mathrm{ft} / \mathrm{s}$, no roll control is required. At speeds greater than $10 \mathrm{ft} / \mathrm{s}$, the allowable error is decreased. This reflects the fact that both crew loads and worst-case structural loads are worse at high horizontal impact speeds. Also note that the shape of the "cloud" of blue error markers reflects the improved navigation accuracy and reduced wind variability at higher wind speeds.

The red requirement boundary in the figure may also be viewed as the system level design "knob" to allow systems engineers to trade the risk of landing in an undesireable orientation, against the mass required to make the structure and crew loads attenuation system robust to larger errors at higher gound speeds. Since the shape of the requirement line is complex we will choose a single driving "choke point" to determine margins and sensitivities. This point is at $40 \mathrm{ft} / \mathrm{s}$ in the figure and the margin is determined by comparing the boundary error at this point $\left( \pm 20^{\circ}\right)$ with three times the standard deviation of the error in the vicinity of the point (for simplicity, we shall assume that the mean error is zero). 
Here the mass of interest is the mechanism and structural mass required to limit crew loads and maintain structural integrity. The sensitivity of interest then is the sensitivity of the heading error at $40 \mathrm{ft} / \mathrm{sec}$ to structural and mechanism mass. Notional values of the margin and sensitivity are provided in Table 4.

Table 4. Margin and Sensitivity for Heading Error Trade

\begin{tabular}{|l|c|c|c|}
\hline Trade & Requirement/ Design Objective & Margin & Sensitivity (\%/lb) \\
\hline $\begin{array}{l}\text { Heading error performance vs. } \\
\text { Structural Mass }\end{array}$ & $\begin{array}{l}3 * \sigma \text { of heading error }<20 \text { deg at } 40 \\
\mathrm{ft} / \mathrm{sec}\end{array}$ & $15 \%$ & 0.33 \\
\hline
\end{tabular}

\section{Vehicle Design Application and Lessons Learned}

The studies summarized above provided information to the Orion SEIT team and Orion mana gement to make vehicle-level design decisions which are still in process at the time of this writing. This section looks at a process for combining the study results to make design decisions, and provides lessons learned during the conduct of these and other studies. It is important for the analyst to bear in mind the purpose of the analysis since the information must be presented to management in a clear manner, and should include the underlying physics (at a high level), the applicable requirements, the margin on those requirements and the sensitivity of that margin to mass.

\section{A. Margin Balancing}

For the purposes of illustrating some of the elements of a decision making process, we have simplified the study results somewhat so that a classical margin balancing technique may be used to allocate mass between vehicle components. After discussing these process elements, considerations for real world application will be covered.

Table 5 summarizes the data and results of four of the studies presented above. These four studies had attributes that lend themselves well to mathematical margin balancing, namely they relate mass to a required performance or safety metric, and their results provided the margins and sensitivities required to balance margin. In the following discussions, we refer to mass as the "currency" used to balance margin. Since we have a fixed amount of currency (mass) in Orion, the systems engineering decision is how to allocate, or "spend" the currency to optimize performance.

Table 5. Margin Balancing for Mass Allocation

\begin{tabular}{|l|l|l|c|c|c|c|}
\hline Study & Trade & $\begin{array}{l}\text { Requirement/ } \\
\text { Design Objective }\end{array}$ & Margin & $\begin{array}{l}\text { Sensitivity } \\
(\% / \mathrm{lb})\end{array}$ & $\begin{array}{c}\Delta \mathrm{m} \text { for } \\
\text { Margin } \\
\text { Balance (lb) }\end{array}$ & $\begin{array}{c}\text { Balanced } \\
\text { Margin }\end{array}$ \\
\hline LAS Ballast & $\begin{array}{l}\text { Success vs. } \\
\text { Ballast Mass }\end{array}$ & $\begin{array}{l}80 \% \text { Success at any } \\
\text { altitude }\end{array}$ & $-19 \%$ & 0.23 & +134.9 & $12.0 \%$ \\
\hline $\begin{array}{l}\text { Lunar Surface } \\
\text { Coverage }\end{array}$ & $\begin{array}{l}\text { Surface Coverage } \\
\text { vs. Propellant } \\
\text { Mass }\end{array}$ & $\begin{array}{l}65 \% \text { of lunar surface } \\
\text { acheiveable on } \\
100 \% \text { of launch } \\
\text { epochs }\end{array}$ & $20 \%$ & 0.12 & -66.4 & $12.0 \%$ \\
\hline $\begin{array}{l}\text { Entry } \\
\text { Performance }\end{array}$ & $\begin{array}{l}\text { L/D vs Ballast } \\
\text { Mass }\end{array}$ & $\begin{array}{l}\text { Mean L/D }-3 * \sigma \text { of } \\
\text { L/D }>=0.23 \text { (lunar) }\end{array}$ & $15 \%$ & 0.05 & -59.5 & $12.0 \%$ \\
\hline $\begin{array}{l}\text { Touchdown } \\
\text { Heading } \\
\text { Control }\end{array}$ & $\begin{array}{l}\text { Heading error } \\
\text { performance vs. } \\
\text { Structural Mass }\end{array}$ & $\begin{array}{l}3^{*} \sigma \text { of heading } \\
\text { error }<20 \text { deg }\end{array}$ & $15 \%$ & 0.33 & -9.0 & $12.0 \%$ \\
\hline
\end{tabular}

Recall that the ballast margin for the LAS is not lifted all the way to orbit as discussed above, so the ballast mass delta shown in the table is multiplied approximately by 8 to arrive at the actual ballast to be housed in the LAS - or around $1000 \mathrm{lb}$.

Once the system level designer has the information of margin and sensitivity to each requirement with respect to a common currency, he should balance the margin. This may be accomplished by one of these methods:

1. Modify the requirements: It is always a good idea to revisit the relative importance of the various requirements during margin balancing. How important is the abort success rate compared to Lunar surface coverage given abort probabilities and mission objectives? These kinds of decisions must be 
made at a project level and the role of the Flight Dynamics team and other engineering organizations is to provide accurate, relevant and digestable data to aid in the decision.

2. Re-allocate the currency: A mathematical example of this process is illustrated in the right two columns of the table and the equations which were applied are derieved in the appendix from the straight forward assumptions that the margin must be balanced with zero change in mass and after balancing, the margins are equal on all requirements.

3. Apply management reserve: The assumption that the mass change must be zero assumes that no mass growth is allowed. As the design matures, management may opt to release mass margin in the form of reduced weight growth allowance or reduced management reserve. This will allow improved performance across the flight regimes in the table, but the margin balancing principals remain the same.

4. A combination of the above: Systems engineers may elect to modify requirements, re-allocate mass and apply reserve.

The Orion systems engineering problem is much more complex that the example presented. There are many more performance metrics, precise sensitivities are often difficult to determine, and mass on the SM is not equivalent to mass on the CM since they drive different performance metrics. In practice, systems engineers combine classical margin balancing with engineering judgement, but systems engineers should always know their margins, understand sensitivities, and ensure that no margins stand out.

The last of these is the crux of the vehicle-level systems engineers role. The role of the flight dyanamics analyst, on the other hand, is to provide data on margins and sensitivities. The next section discusses some lessons gleaned from these and other flight dyanamics studies which were conducted with the intent of supplying data to make vehicle-level decisions.

\section{B. Use of Time Domain Simulation and Monte Carlo Analysis in Trade Studies}

All of the trade studies described above used time domain simulation to produce the trade results. Time domain simulations techniques have become more and more popular over the last few decades to generate data for vehicle design. Monte Carlo analysis is often used to derive statistical correlations and sensitivities as shown above. This approach has some benefits, including:

- The process of simulation model development and integration often provides experience and insight to the developer into the governing physics and performance drivers in applicable flight phases (but this may not always be true for the analyst who may not have been involved in model development)

- Modern computer systems are capable of handling hundreds of parameter variations over thousands of simulation runs. These can provide accurate Monte Carlo statistics over the parameter ranges used for the models used

On Orion, Monte Carlo analysis will be used extensively for requirements verification, but it has also been used for requirements development, sensitivity analysis, and vehicle-level design. Design recommendations gleaned from these applications include:

- Understand the underlying physics

- Use Monte Carlo (or other statistical approaches) sparingly in requirements allocation or error budgeting

- Understand when worst-case analysis is conservative and when it is appropriate

- Avoid pitfalls in Monte Carlo analysis for requirements verification

The following paragraphs details these recommendations.

\section{Recommendation: Understand the underlying physics}

The first recommendation is well known, but bears repeating. When new, inexperienced engineers join a project, they are often assigned simulation analysis tasks. These "turn the crank" jobs can allow engineers to begin producing results soon after joining an organization. However, it takes discipline on the part of supervisory personnel to make sure that the simulations are configured correctly, that the statistical attributes of Monte Carlo parameters are properly set, that output statistics are correctly interpreted, and that the analyst develops a knowledge of the underlying physics. We will discuss some of the statistical aspects below, but the system knowledge can only be gained by accompanying the simulation study with theoretical analysis. One rule of thumb: "never be surprised by a simulation result." That is to say that the simulation or Monte Carlo analysis should confirm what the analyst 
expects or should refine the analysts' estimate. When this is not the case, the analyst must understand why the results do not match expectations. The sim can be wrong!

\section{Recommendation: Use Monte Carlo sparingly in requirements allocation or error budgeting}

Figure 15 is a simple diagram of the Monte Carlo process which will aid in discussing lessons learned. In the process, the statistical properties of the input parameters are defined, then random values are selected for each of the parameters, and a simulation (or other process) is executed using these values. The resulting parameters of interest are recorded, and the process repeated many times. The statistical properties of the output provides information that may be used to decompose requirements, serve as an input for other design processes or to verify existing requirements.

As an example of requirements decomposition, consider the heading error example in the roll orientation trade above. For this example the requirement on the GNC substystem was "three times the standard deviation of the heading error (assuming zero mean) at $40 \mathrm{ft} / \mathrm{sec}$ groundspeed shall be less than $20^{\circ}$." One of the jobs of the GNC

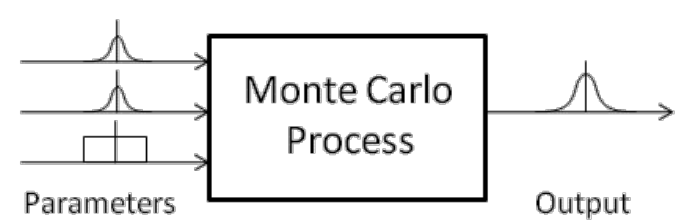

Figure 15. Monte Carlo Process system designer is to decompose this requirement into navigation accuracy and control error allocations. In this example the total error is just the sum of the navigation error and the control error. Here, control error includes error due to disturbances - such as wind gusts wind shear.

One familiar approach to allocating error to navigation and control is the worst case approach. The worst case may be defined as a hard limit, or it may be defined by a project policy such as "design to three sigma." Either way, the total error is the sum of the worst case navigation and control errors. If the total error meets the requirement, then a portion of the margin may be allocated to the lower level requirements in proportion to their contribution. This method is the most conservative method of performing allocation since total error is highly unlikely to exceed the sum of the worst case errors. It is also the simplest method.

If the total error does not meet the requirement, then the systems engineer may elect to petition for a more generous requirement (at a structural mass cost), try to improve navigation and or control performance (perhaps at a cost of more expensive hardware or more complex software) - or he may elect to use a less conservative allocation process. One method would be to assume that the total error distribution is a statistical combination of the input distributions. If both navigation and control error are random with Gaussian distribution, then the distribution of the sum of the errors is Gaussian with standard deviation equal to the root-mean-square (RMS) of the inputs.

If the Gaussian statistical assumptions are not applicable to the inputs, or if the relationship between inputs and outputs is complex, then, Monte Carlo analysis is sometimes used to relate the statistical properties of the inputs to the outputs. However, if Monte Carlo is used, two pitfalls must be avoided:

- The output distribution must be close enough to Gaussian to meet the intent of the requirement

- Margin must be applied during requirements allocation

The later pitfall is the most common and causes the most difficulty. If the lower level requirements (navigation and control error specifications in the example) are simply left at their expected " $3 \sigma$ " values, and if there is insufficient margin at the next level (total heading error in the example), then any change in applicable parameters or assumptions may cause an increase in navigation or control variances, which may in turn cause an exceedance of the heading error requirement, which may necessitate redefinition of the requirement, which can affect structural mass and ultimately require re-allocation of mass at the vehicle level. In our example, if the landing site changes, and the wind shear statistics worsen, then the control variances will increase, and the effect can propagate up the requirements chain. To prevent this, margin should be consciously allocated early through all requirements levels.

\section{Recommendation: Understand when worst-case analysis is conservative and when it is appropriate}

As mentioned above, worst case analysis is a simple approach to requirement decomposition and includes a level of conservatism or "built in" margin. But analysts often confuse the low probabilityof a worst case combination with conservatism. To illustrate this, consider Figure 16 - an abstraction of the input parameter space. The horizontal axis represents the value of an input parameter, "p1" and the vertical axis another parameter, "p2." The mean values of $\mathrm{p} 1$ and $\mathrm{p} 2$ are shown by the cross at center, while the red box represents the range of values between mean minus $3 \sigma$ and mean plus $3 \sigma$. The green ellipse represents the region in the $\mathrm{p} 1, \mathrm{p} 2$ parameter space for which a requirement is met. 


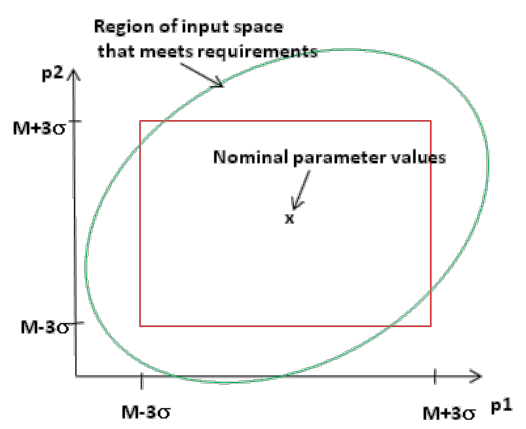

Figure 16. Parameter Box
The common argument for using a statistical approach to reduce conservatism is to note that the probablitly of the p1, p2 pair existing near the corners of the box is low. [Include equation here. I think it's $\left.(1-99.73)^{\wedge} \mathrm{n}\right]$. As the number of parameters increases, the probability of parameters combining at the corners of the box in a "worst on worst" combination rapidly approaches zero. So assessing to the corners of the box that cause requirements exceedance may be unnecessarily conservative.

While it is true that worst case analysis assumes a low probability condition, the degree to which it is conservative depends on the relative sensitivity of the output to the parameters. The left diagram of Figure 17 shows a case where $\mathrm{p} 1$ is a "driving" parameter and the output is relatively insensitive to $\mathrm{p} 2$. In this case, evaluating the corner of the box does not add a significant amount of conservatism - even though the p1, p2 pair at the corner is highly improbable. And the same is true for the right hand diagram, in which the output is not highly sensitive to either parameter in the context of their variances.

It is often the case that a system response is driven largely by one or two parameters, so, depending on the amount of margin available, and the relative sensitivity of the output to the other parameters, a worst case, or "corners of the box" approach to requirement

decomposition is often the simplest and most robust method.

This worst case approach was used by the Orion flight dynamics team in defining the requirements for CG offset as discussed in the L/D section above. For missions to ISS, the CG offset was derived by assuming that aerodynamic parameters were at their worst values (when uniformly distributed) or their $3 \sigma$ values when Gaussian. This allowed a simple definition for a CG box with adequate margin for uncertainties and operational variations in CG. For the Lunar mission, however, margin was much more constrained - largely by a higher minimum L/D requirement, so a more sophisticated statistical approach was taken, as described in reference XXX.

\section{Recommendation: Avoid pitfalls in Monte Carlo analysis for requirements verification}

As the project approaches CDR, the requirements allocation process is largely complete, and the focus shifts toward proving requirements compliance via pre-CDR verification. During this phase, simulation analysis using higher fidelity models is an appropriate method for assessing requirements compliance. However, several common pitfalls should be avoided:

1. Set parameters according to bounding requirements - not expected values

2. Margin is determined from output results - not input variations

3. ... however, input variations can and should be used to determine robustness - find out where it breaks

4. TBS

\section{Conclusions}

TBS

\section{Appendix}

\section{A. Margin Balancing Equations}

Once margin and sensitivity data are available for each requirement, the equations to re-allocate the independent cost variable (often mass, or monetary cost, or a combination) may be derived as follows:

The change in margin for the $\mathrm{i}^{\text {th }}$ requirement, $\Delta M_{i}$ is added to the existing margin to achieve the balanced margin which we desire to be constant for all requirements: 
The change in margin may be replaced by the change in cost variable, $C$ times the sensitivity of margin to the cost variable:

so

and

Now, when re-allocating cost, the net cost change between all n requirements must be zero:

$$
=0
$$

so $=0$

This expression contains the constant balanced margin for each requirement, $M_{b}$, and the know values for the margins and sensitivities for each requirement. To solve for $M_{b}$, the common denominator for each of the terms in the sum is multiplied by each sum:

Define:

so

so the desired balanced margin value is:

and the changes in cost variable and margin for each requirement are may be determined from 1 , and 2 above.

\section{B. Margin for Statistical Success Requirements}

The approach taken in this exercise was to presume that abort failure is the result of an "underlying performance parameter" that exceeds a limit. We further assume that the underlying parameter has a Gaussian distribution of mean zero and a standard deviation that causes the limit to be exceeded $5 \%$ of the time. This standard deviation then is the required standard deviation of the parameter. We then compute the standard deviation that causes the parameter to exceed the limit at the observed percentage. Margin is then computed as the difference between these standard deviations.

TBS.

\section{Acknowledgments}

Mike Hughes

AAMT leads and analysts

Trajectory leads and analysts (Condon, Williams et. al.)

EMT leads and analysts

\section{References}

The.

Jeremy Rea’s paper 
Condon's lunar coverage analysis (tech brief?)

Jackson's TEI presentation

Condon, Williams et al TEI tech brief?

LAV Ballast TDS?

Periodicals

${ }^{1}$ Vatistas, G. H., Lin, S., 


\title{
Orion Flight Performance Design Trades
}

\author{
Mark C. Jackson ${ }^{1}$ \\ Charles Stark Draper Laboratory, Houston, TX, 77062 \\ and \\ Timothy Straube ${ }^{2}$ \\ NASA Johnson Space Center, Houston, TX, 77058
}

\begin{abstract}
A significant portion of the Orion pre-PDR design effort has focused on balancing mass with performance. High level performance metrics include abort success rates, lunar surface coverage, landing accuracy and touchdown loads. These metrics may be converted to parameters that affect mass, such as ballast for stabilizing the abort vehicle, propellant to achieve increased lunar coverage or extended missions, or ballast to increase the lift-to-drag ratio to improve entry and landing performance. The Orion Flight Dynamics team was tasked to perform analyses to evaluate many of these trades. These analyses not only provide insight into the physics of each particular trade but, in aggregate, they illustrate the processes used by Orion to balance performance and mass margins, and thereby make design decisions. Lessons learned can be gleaned from a review of these studies which will be useful to other spacecraft system designers. These lessons fall into several categories, including: appropriate application of Monte Carlo analysis in design trades, managing margin in a highly mass-constrained environment, and the use of requirements to balance margin between subsystems and components. This paper provides a review of some of the trades and analyses conducted by the Flight Dynamics team, as well as systems engineering lessons learned.
\end{abstract}

$\begin{array}{ll}\text { ACM } & =\text { Attitude Control Motor } \\ \text { CEV } & =\text { Crew Exploration Vehicle } \\ \text { CG } & =\text { Center of Gravity } \\ \text { CM } & =\text { Crew Module } \\ \text { GN\&C } & =\text { Guidance Navigation and Control } \\ \text { GPS } & =\text { Global Positioning System } \\ \text { LAS } & =\text { Launch Abort System } \\ \text { LAV } & =\text { Launch Abort Vehicle } \\ \text { LLO } & =\text { Low Lunar Orbit } \\ \text { OME } & =\text { Orion Main Engine }\end{array}$

$\begin{array}{ll}\begin{array}{c}\text { Nomenclature } \\ \text { RMS }\end{array} & =\text { Root Mean Square } \\ \text { SM } & =\text { Service Module } \\ \text { TCM } & =\text { Trajectory Correction Maneuver } \\ \text { TEI } & =\text { Trans Earth Injection } \\ \text { TLI } & =\text { Trans-lunar Injection } \\ & \\ \Delta V & =\text { Change in inertial velocity } \\ I_{s p} & =\text { Specific Impulse } \\ L / D & =\text { Lift to Drag Ratio } \\ V_{r e l} & =\text { Earth Relative Velocity }\end{array}$

\section{Introduction}

$\mathrm{T}$ The Orion Crew Exploration Vehicle (CEV) consists of three major components (Figure 1): a Crew Module (CM), Service Module (SM) and a Launch Abort System (LAS). The requirement for Orion to carry four crew members to and from the Moon has resulted in a larger, heavier CM than the Apollo design. The capsule diameter is $16.5 \mathrm{ft}$, nearly $4 \mathrm{ft}$ larger than the Apollo capsule with 2.5 times the internal volume. This larger capsule requires a larger, more powerful LAS to achieve required separation during launch aborts. The SM, on the other hand is smaller than its Apollo counterpart, since the task of inserting the vehicle into lunar orbit falls to the lunar lander

\footnotetext{
${ }^{1}$ Lead Systems Engineer, Orion Flight Dynamics Team, NASA Johnson Space Center, AIAA Member.

${ }^{2}$ Orion Flight Dynamics Team Lead, Aerosciences and Flight Mechanics Division, NASA Johnson Space Center, AIAA Member.
} 
propulsion system, rather than the SM engine, as in Apollo. Even with the smaller service module though, mass has proven to be a critical design parameter for Orion. The mass of the Orion CM affects abort system performance, landing loads, parachute sizing, launch vehicle performance and trans-lunar injection (TLI) performance. The mass of the SM drives the range of lunar surface sites that are achievable at a given Earth-Moon geometry as well as launch and TLI performance.

A significant portion of the Orion pre-PDR design effort has focused on balancing mass with performance. High level performance metrics include abort success rates, lunar surface coverage, mission duration

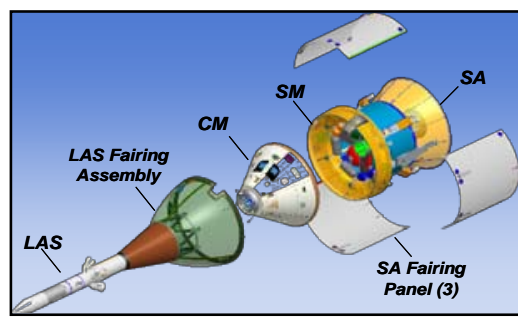

Figure 1. Orion Components limits, landing opportunity rates and landing accuracy. These metrics may be converted to parameters that affect mass, such as ballast for stabilizing the abort vehicle, propellant to achieve increased lunar coverage or extended missions, or ballast to increase the lift-to-drag ratio to improve entry and landing performance. The Orion Flight Dynamics team was tasked to perform analyses to evaluate many of these trades. These analyses not only provide insight into the physics of each particular trade but, in aggregate, they illustrate the processes used by Orion to balance performance and mass margins, and thereby make design decisions. Lessons learned can be gleaned from a review of these studies which will be useful to other spacecraft system designers. These lessons fall into several categories, including: appropriate application of Monte Carlo analysis in design trades, managing margin in a highly mass-constrained environment, and the use of requirements to balance margin between subsystems. This paper provides a review of some of the trades conducted by the Flight Dynamics team.

The following sections describe selected trade studies whose results quantified the sensitivities of vehicle performance to mass. Trade study descriptions are divided into ascent abort, orbit, and entry sections. The ascent abort section covers a trade on the stability of the abort vehicle versus ballast mass. Orbit trades selected are "fail safe" Moon-to-Earth transfer maneuvers and Lunar coverage studies. Entry studies include entry performance versus ballast mass, and active roll control performance under chutes to orient the vehicle at touchdown. Each trade study contains a summary of the results in terms of performance margin and sensitivity of performance to mass.

After the trade study discussions we use the margin and sensitivity data to provide a notional example of the margin balancing that is performed at the Orion project level. The example highlights many of the issues that must be dealt with by Orion systems engineers and provides a conceptual framework for using the study information

The final sections present lessons learned from the conduct of the studies and from the application of their results in vehicle-level integration. These include lessons on simulation and Monte Carlo analysis - from avoiding traps during the analysis to interpreting the results correctly.

\section{Definition of Margin and Sensitivity}

To aid in better defining margin and sensitivity for the rest of this paper, it is useful to define a relative performance metric. This metric is the measured performance, divided by the performance requirement. Using landing accuracy as an example, if a study shows that 3 times the standard deviation $(3 \sigma)$ of landing accuracy for a particular set of parameters is 4 nautical miles, and the required landing accuracy $(3 \sigma)$ is $5 \mathrm{~nm}$, then the relative performance metric is 0.8 - so this relative metric equals one when the performance equals the requirement. Margin then, is one minus the relative performance metric. In the landing example, margin is 0.2 or $20 \%$.

Sensitivity is normally thought of as the partial derivative of the performance metric to a cost variable. The cost variable used here is mass, but other cost metrics are possible, including monetary cost. In the landing example, if adding $100 \mathrm{lb}$ of ballast to the $\mathrm{CM}$ increases landing accuracy by 0.2 nautical miles, then the sensitivity of accuracy to mass is $0.2 / 100$ or $0.002 \mathrm{~nm} / \mathrm{lb}$. This type of sensitivity however does not allow the systems engineer to trade different types of performance. Which is worth more, for example, landing accuracy or lunar surface coverage?

The measure of "importance" of a particular performance metric is the requirement. So for comparison to other performance metrics, we want the sensitivity of margin with respect to the required performance. In the landing example, if the additional mass improves landing accuracy to $3.8 \mathrm{~nm}$, then margin is increased to $22 \%$ and the sensitivity of the margin to mass (in percent) is 100 times the performance sensitivity divided by the requirement, or 0.04. We will use this type of sensitivity (margin to mass) in our examples derived from the trade studies below.

\section{Ascent and Abort Trade Study Example}

During ascent, Orion may abort from a failing, or under-performing launch vehicle in two ways. During first stage flight, a Launch Abort System (LAS) is provided to remove the Crew Module (CM) capsule from a booster experiencing catastrophic failure. Shortly after first stage burnout, the LAS is jettisoned and the responsibility for 
separation from the launch vehicle falls to the Service Module (SM). Second stage booster failures are generally considered more benign that first stage failures. This section describes one of many trades conducted to help design the LAS.

\section{A. Stability vs Ballast}

The Orion Launch Abort System (LAS) consists of three rocket motors housed in a tower-fairing assembly (Figure 2). The abort motor provides the thrust to remove the Launch Abort Vehicle (LAV) - the combined LAS and capsule - from the malfunctioning booster. The jettision motor detaches the LAS from the capsule after it has

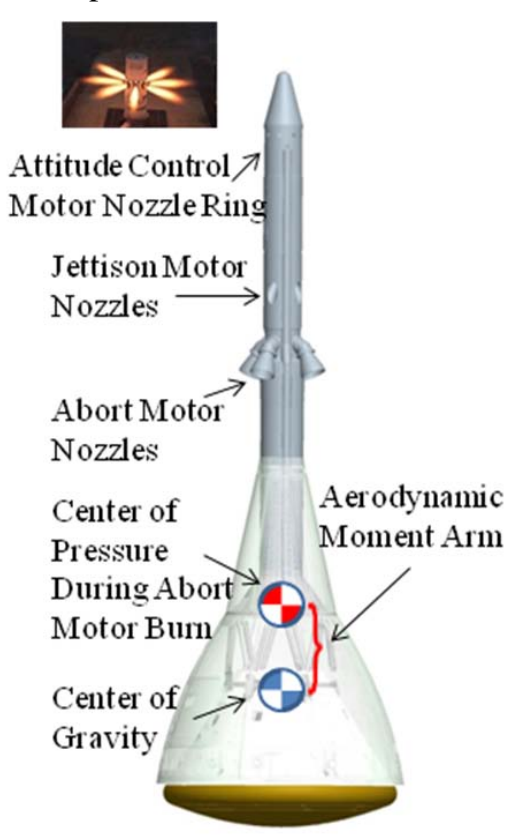

Figure 2. Launch Abort Vehicle been re-oriented to a heat shield forward attitude during the abort. The attitude control motor (ACM) is a solid rocket engine whose thrust is directed through a ring of eight "pintle" valves. The net thrust vector is controlled by opening or closing valves in the ring. The role of the ACM is to stabilize and control the LAV during the rocket motor burn, and to reorient the LAV to the heat-shield forward attitude for LAS jettison and subsequent chute deploy.The Orion abort system is required to prove a $95 \%$ success rate averaged over the ascent time period. A further design objective is to eliminate specific periods during the ascent with low success probabilities.

One of the challenges of controlling the LAV is that the abort motor thrust plumes move the aerodynamic center of pressure ahead of the center of gravity making the LAV dynamically unstable during the abort burn (Figure 3). In addition to the normal challenges associated with un-stable vehicles, the forward center of pressure increases the aerodynamic moment arm for pitch and yaw. This means that large aerodynamic torques may occur when the vehicle is displaced from the trim angle of attack.

The degree to which the abort motor plumes affect the center of pressure is difficult to determine precisely. The resulting uncertainty in pitch moment torque means that under certain conditions the aerodynamic pitch moment may exceed the available torque from the ACM control motor. Should this occur the vehicle will tumble out of control.

The combination of large aerodynamic uncertainties together with increases in the CM mass allocation, resulted in the vehicle tumbling during a significant number of simulated abort cases - especially when aborts occurred around Mach 1. To correct these tumbles, weight or ballast may be added in the top portion of the LAS tower. This moves the center of gravity forward, reducing the aerodynamic moment arm of Figure 2.

To determine the effect of ballast on abort success, a Monte Carlo simulation study was conducted with varying amounts of ballast. Figure 4 summarizes the tumbling failure rates for several quantities of ballast. Aborts were initiated at altitudes between zero (the launch pad) and around $65000 \mathrm{ft}$ - the point at which the LAS is jettisoned after first stage separation. Parameters varied in the Monte Carlo included aerodynamic parameters, mass properties and rocket motor parameters.

The figure shows that with no ballast (blue curve) there are altitude regions in the ascent profile for which the probability of abort failure due to tumbling are relatively high - more than $35 \%$ at the peak and greater than $5 \%$ for a large section of the ascent. The improvements in success rate are clear from the reduced tumbling cases associated with even the relatively "small" $660 \mathrm{lb}$ nose-cone ballast.

The results of this study provided Orion management and systems decision makers data on the success rate as a function of ballast mass. As we shall see, the mass data may be used as a common "currency" to trade first stage abort success performance against other spacecraft attributes which enhace crew safety, improve capability or increase mission success rates. When considering the ballast mass numbers against other sources of mass on the Orion vehicle, bear in mind that, since the LAS is jettisoned after first stage, mass on the LAS is not as critical to ascent performance as other vehicle mass which is fully lifted into orbit. A rule of thumb calculation for comparing LAS mass to CM or SM mass is that one pound of LAS mass affects ascent performance about $1 / 8$ as much as one pound of CM or SM mass. 
In order to balance margins across the vehicle design, we must quantify the margin on vehiclelevel requirements. That presents some difficulty with the success rate requirements that apply to the LAS. For example, consider the LAV requirement of $95 \%$ success. Even if the vehicle is successful $100 \%$ of the time, the margin on this requirement is only $5 \%$ so standard margins of $30 \%$ at PDR and $10 \%$ at CDR are not acheiveable. Further, this limit on maximum margin precludes margin balancing since no amount of ballast assures $100 \%$ success. For these reasons, we have taken the approach of redefining margin based on a fictitious "underlying performance parameter." Failure is presumed to occur when that parameter exceeds a limit. The details of this process are provided in the appendix since, although they are interesting,

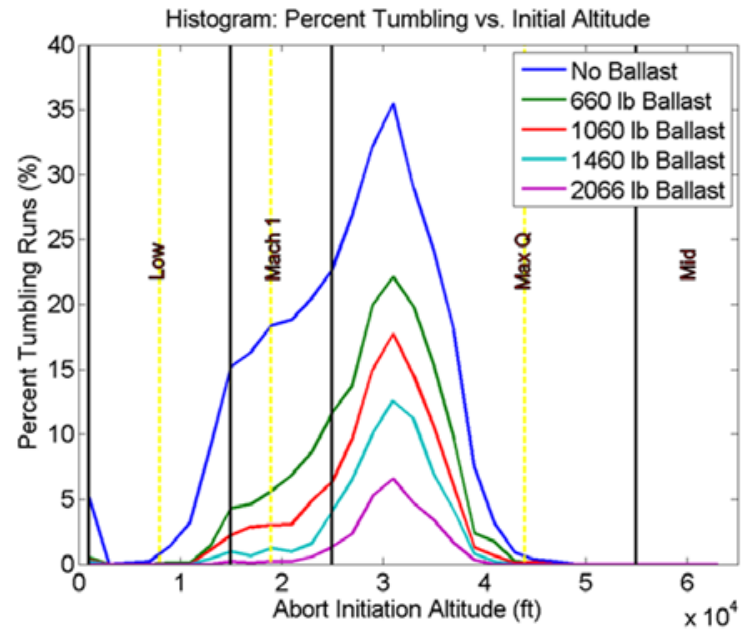

Figure 4. Effect of Ballast on Tumbling they are not germain to the overall process of trading LAS abort performance.

Although the $95 \%$ time-averaged success requirement does not preclude high failure rates at particular points along the profile, the flight dynamics team has the design goal of limiting failure rates along the profile. As of this writing, the vehicle level trades are not complete to determine how much, if any ballast will be allocated. However, for the purposes of the margin balancing discussion below, we will assume that the amount of ballast used should be sufficient to keep success rates above $80 \%$ at all points along the profile. This assumption also simplifies the determination of margin and sensitivity, since we now need only consider the data at the peak failure rate - a little more than 30,000 ft in Figure 4.

Table 1 summarizes the LAV representative performance requirements, margins and sensitivities gleaned from this and similar Monte Carlo studies. Note that with no ballast the LAV has negative margin on the success requirement.

Table 1. Margin and Sensitivity for LAV Ballast Trade

\begin{tabular}{|l|l|c|c|}
\hline Trade & Requirement/ Design Objective & Margin & Sensitivity (\%/lb) \\
\hline Success vs. Ballast Mass & $80 \%$ Success at any altitude & $-19 \%$ & 0.23 \\
\hline
\end{tabular}

Of course, other parameters affect performance as well as ballast. Expected performance may be improved by reducing aerodynamic uncertainties through flight or wind-tunnel tests, by improving the control design, or by any number of other techniques. But as Orion approaches CDR the requirements, control design and major environmental models will become more stable, and final decisions will be made to allocate mass according to the remaining vehicle-level "knobs" such as ballast and propellant.

\section{Orbit Trades}

This section presents two trade studies conducted to relate Orion performance to SM propellant mass. The first study looked at the techniques used in targeting and executing main engine burns at the moon. This study was used to determine the assumptions that should be used in generating propellant budgets for lunar missions. The second study determined available lunar surface coverage as a function of SM propellant.

\section{A. Fail Safe Trans Earth Injection}

During the lunar mission, after the Altair lander has returned the crew to Orion in lunar orbit, the Altair ascent stage is jettisioned, and the Orion SM performs the series of burns at the Moon to initiate the orbital transfer back to Earth. A typical three burn, trans-Earth injection (TEI) series is depicted in Figure 5. TEI-1 transfers Orion from a near circular low lunar orbit (LLO) to a highly elliptical orbit (yellow arc). Near the apoapse of this orbit, TEI-2 is executed to place the vehicle in a lunar orbit plane that corresponds to the plane of the desired Earth-relative velocity for the return trip. TEI-3 is then executed to accelerate away from the Moon and enter an Earth-relative orbit with 
the desired periapse for Entry. On the return trip, trajectory correction maneuvers (TCMs) are executed to correct dispersions from the ideal TEI sequence and to precisely target the entry interface conditions.

During the TEI sequence, two types of failures can cause the spacecraft to impact the Moon: 1) failure to execute a burn - which can cause impact if the current orbit has a perigee below the lunar surface or, 2) an engine failure during a burn - which can cause impact if the resulting orbit has a perigee below the surface.

The first of these failures - failure to execute - results in impact if the previous burn left the spacecraft in an orbit with a low perigee. This is a function of how the previous burn is targeted and can occur if, for example, the burn had a radial component. Experience has shown that a fuel optimal burn sequence can result in orbits with unsafe periapse. Figure 6 shows an example in which TEI-1 is executed to target the optimal TEI-2 position - resulting in an orbit that impacts the lunar surface if TEI-2 is not executed. As the figure shows, from the time that TEI-2 fails to execute $(t=0)$ to lunar surface impact is 12.2 hours. For this particular failure then, some time remains to correct the problem, prevent lunar impact, and re-target the return.

This situation may be contrasted with that of Figure 7. In this example, TEI-2 is executed normaly, but the optimal solution leaves Orion in a lunar impact orbit. This solution relies on TEI-3 executing properly to prevent collision with the Moon, and - as the figure shows only leaves about 12 minutes to correct a problem once the failure is discovered at TEI-3 execution.

The collisions depicted in Figures 6 and 7 may be prevented by adopting a policy constraining the targeting of all burns to result in periapse above a prescribed altitude. This policy is referred to here as "fail safe targetting."

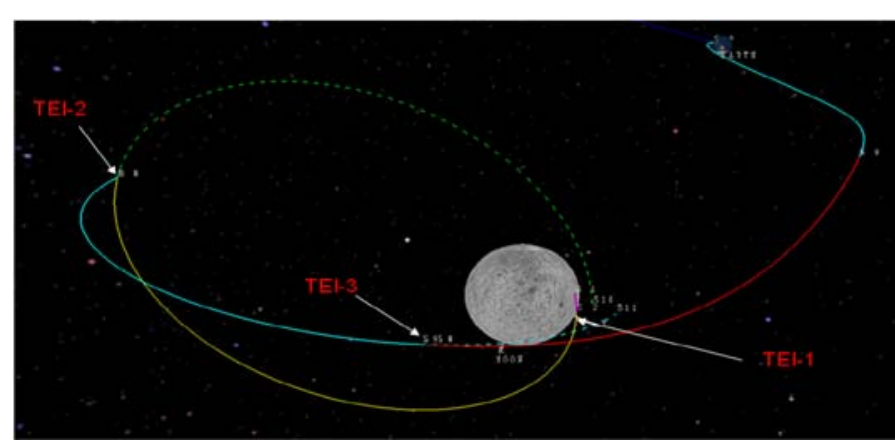

Figure 5. Trans-Earth Injection (TEI) Sequence

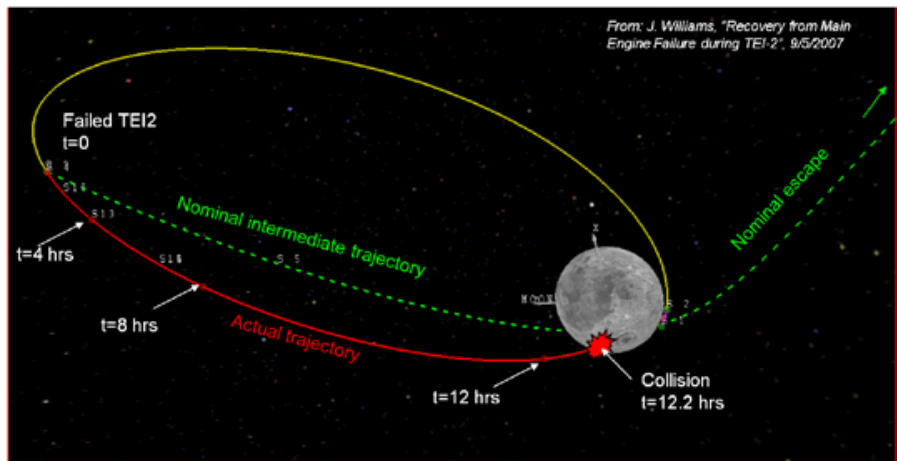

Figure 6. Impact Resulting from Failed Burn Execution

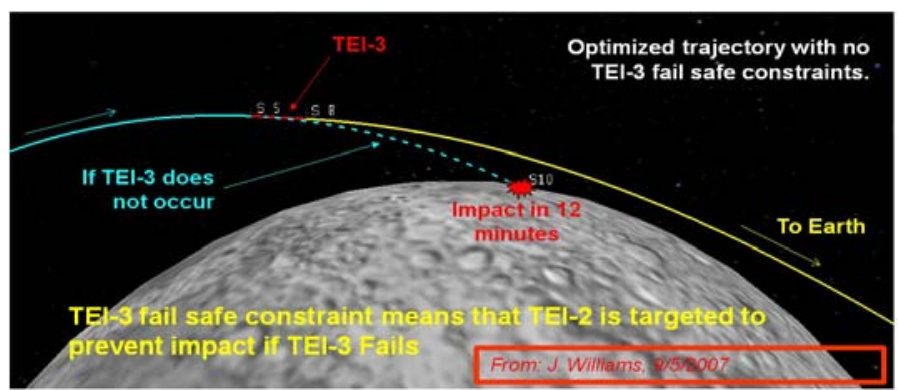

Figure 7. Impact Resulting from Failed TEI-3 Execution

The second type of failure considered is an engine failure during the burn. Figure 8 is a conceptual diagram of the velocity change executed at TEI-2. In this example, the velocity direction is changed by $90^{\circ}$ at or near apoapse, resulting in an orbit whose plane is consistent with the orbit plane needed for Earth return. The red traces in the

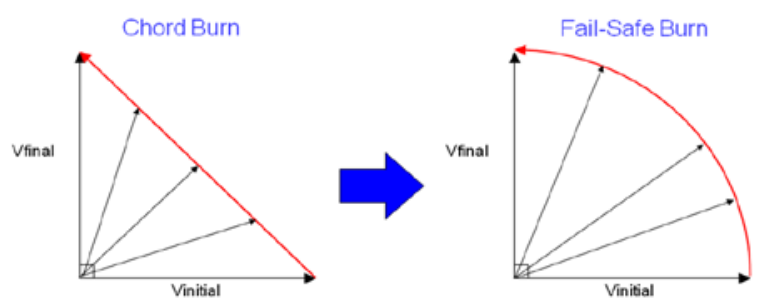

Figure 8. Fail Safe Burn Geometry figure represent two candidate burn execution strategies. On the left the "chord burn" is executed in the direction to most efficiently and directly change the velocity from the intial orbital velocity to the final desired. However, if the burn should stop for any reason along the red trace, the resulting velocity magnitude is less than the initial and final velocities. This smaller velocity magnitude may result in an orbit with lower periapse which impacts the moon.

In contrast, the fail safe burn is executed so that the 
resulting velocity magnitude always results in a safe orbit - at the cost of the extra propellant corresponding to the longer red arc.

The fail safe TEI trade study quantified the propellant mass costs for both fail safe targeting and fail safe execution. Propellant numbers are omitted here since spacecraft performance may be sensitive, but the study recommendations may be understood from the above discussion. They were:

- TEI-1 should be targeted to prevent low periapsis since the fuel penalty was always very small

- TEI-2 execution is not required to be fail safe since many hours are available to correct the resulting orbit, and since auxiliary thrusters are available to back up the Orion main engine (OME)

- TEI-2 is targeted to guarantee a safe periapsis since little time remains after a TEI-3 failure

This trade study did not quantify margin with respect to a requirement. Rather, it resulted in a policy for use in other design trades, such as the following lunar suface coverage trade study.

\section{B. Lunar Geographic and Temporal Coverage}

The Orion concept of operations allows for two types of lunar missions. Lunar outpost missions are intended to service a lunar base at the Moon's south pole. During outpost missions, Orion loiters unmanned in LLO for up to six months. Lunar sortie missions are intended to allow exploration of any portion of the lunar surface and are much shorter than lunar outpost missions - lasting around 18 days under nominal conditions with a week on the lunar surface.

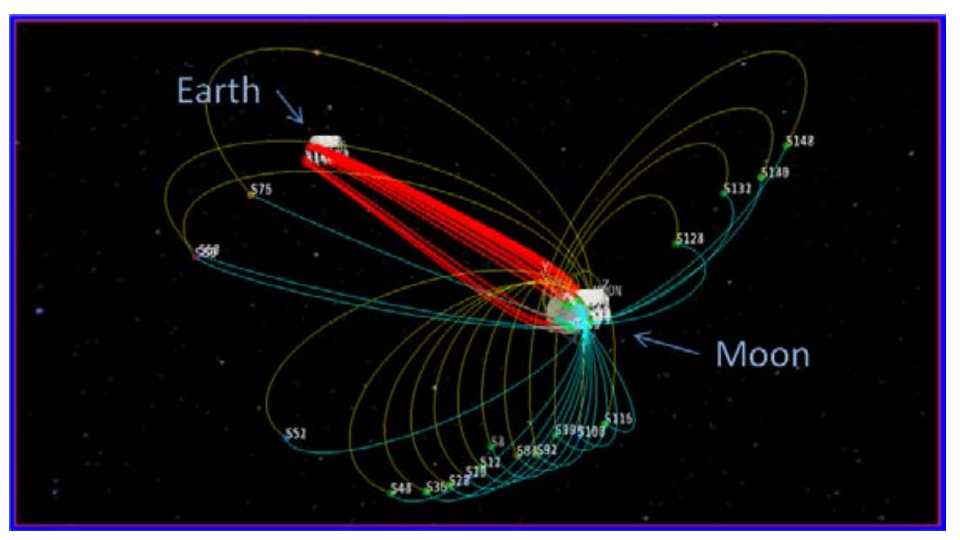

Figure 9. Return Trajectories from Assorted Landing Site
In order to size Orion propellant loads and tank capacities, it is useful to relate the amount of the lunar surface that can be reached to propellant mass. To this end, it is useful to define two types of lunar coverage: geographic coverage, and temporal coverage. Geographic coverage is the amount of the Moon's surface that may be reached at a given launch epoch while temporal coverage is the percentage of time (over the 19 year Metonic cycle) that a particular site can be reached. Given prescribed launch vehicle and lander capacities, the reachable portions of the Moon are determined by Orion's capacity to change it's lunar orbit plane to pass over the landing site, and then reach an appropriate entry interface condition via the three burn TEI sequence described above. This capacity depends on the total inertial velocity change, $\Delta \mathrm{v}$, that Orion can produce, which in turn depends on the specific impulse $\left(\mathrm{I}_{\mathrm{sp}}\right)$ of the engines, and the amount of onboard propellant.

Figure 9 shows the TEI sequences and return trajectories from several landing sites at constant latitude and varying longitude around the Moon. From the figure, it is clear that the TEI burn sequence associated with a particular site has a large effect on the total $\Delta \mathrm{v}$ required to return from that site. So if the propellant load is smaller than that required for the worst of these trajectories, then some percentage of these sites will not be available for exploration at a given epoch.

Figure 10 summarizes the results of a study conducted to determine site availability as a function of propellant load. The study developed mission designs (sequences of burns) for a grid of landing sites around the Moon across a set of departure epochs that spanned the Metonic cycle which governs Earth-Moon geometry. The study consisted of nearly 1 billion site-epoch combinations. 
The horizontal axis of the figure is the onboard propellant in kilograms and the vertical axis shows the percentage of the lunar surface that may be reached given a particular propellant load and a desired amount of temporal coverage. The curves on the graph are the temporal coverage available at a given combination of propellant load and surface coverage.

To read the graph, start with a desired propellant load - say the usable propellant load indicated by the thick red vertical line. Follow the line up to a desired temporal coverage curve - say $90 \%$ temporal coverage. The intersection of the red propellant line with the $90 \%$ curve occurs at about $80 \%$ surface coverage. This means that with around $7400 \mathrm{Kg}$ of useable propellant, Orion can reach $80 \%$ of

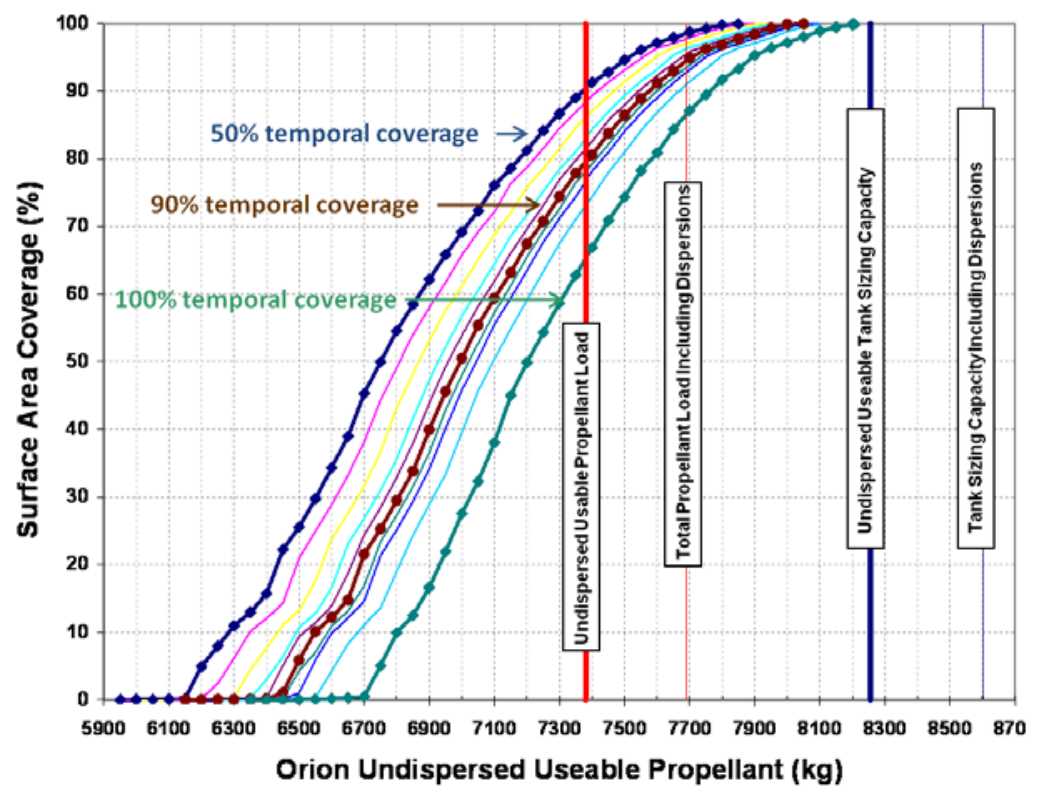

Figure 10. Geographic and Temporal Coverage as a Function of Propellant Load

the Moon's surface $90 \%$ of the time. For the same propellant load, Orion can reach about $65 \%$ of the surface $100 \%$ of the time.

The solid red boundary in the figure is the total propellant required to ideally actuate the mission $\Delta \mathrm{v}$ assuming no targeting, navigation or control errors. Propellant must be added to the ideal value to account for these errors, or dispersions, in order to fly the real mission. This additional propellant is indicated by the thin red boundary in the figure. Margin may also be added to the total propellant load to account for uncertainties or contingencies. The thin red boundary may be thought of as the "design fuel load" since it represents the amount of fuel that is accounted for when trading propellant mass against other forms of mass (strucuture, ballast, heat shield, crew supplies, etc). The design fuel load may be viewed as a requirement which may be adjusted by systems engineers to balance margin.

For our purposes, we will consider the $100 \%$ temporal coverage of $65 \%$ of the surface to be the propellant defining requirement. Recall that the corresponding propellant also provides considerably more surface coverage during more favorable ephocs. Table 2 contains notional values (actual values may be sensitive) of the margin that the design propellant load contains. The sensitivity of surface coverage margin to propellant mass is also provided.

Table 2. Margin and Sensitivity for Lunar Surface Coverage Trade

\begin{tabular}{|l|l|c|c|}
\hline Trade & Requirement/ Design Objective & Margin & Sensitivity (\%/lb) \\
\hline $\begin{array}{l}\text { Surface Coverage vs. Propellant } \\
\text { Mass }\end{array}$ & $\begin{array}{l}65 \% \text { of lunar surface acheiveable on } \\
100 \% \text { of launch epochs }\end{array}$ & $20 \%$ & 0.12 \\
\hline
\end{tabular}

Finally, we note that the thin blue boundary in the Figure 10 represents the total propellant available in the SM tanks. Tank capacity exceeds the design fuel load to provide operational flexibility and future performance growth should other mass opportunities manifest themselves. Note that the dispersions must also be subtracted from the tank sizing capacity to get the "useable" fuel that may be ideally converted to $\Delta \mathrm{v}$.

\section{Entry Trades}

Two trades from the entry flight phase further illustrate the methods used to relate performance to mass. The first relates the capsule lift-to-drag ratio to ballast mass, and the second trades touchdown landing performance with structural mass.

\section{A. Entry Performance vs Ballast Mass}


After entering the atmosphere, the Orion CM seeks a "trim" attitude that balances aerodynamic torques. Figure 11 diagrams some of the parameters which determine the pitch trim angle. In the figure, $\alpha$, or angle of attack is the

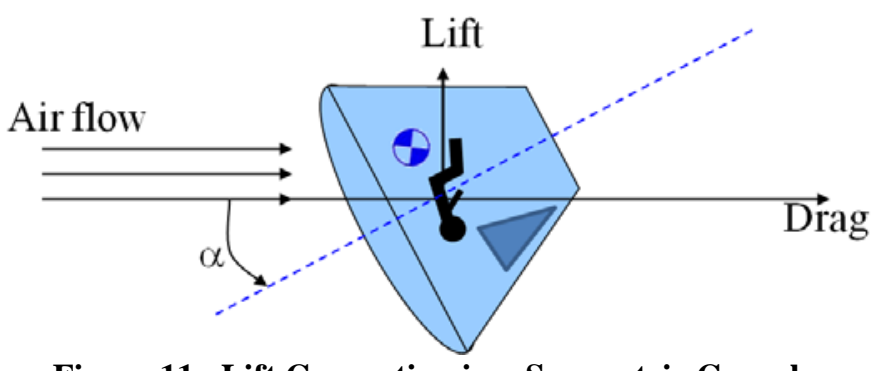

Figure 11. Lift Generation in a Symmetric Capsule angle between the relative air flow and the longitudinal axis of the vehicle (blue dashed line). At small angles of attack, the aerodynamic center of pressure on the CM is close to the center of the heat shield. When the center of gravity (CG) is offset from the longitudinal axis, a torque is generated about the CG that equals the aerodynamic force times the moment arm from the longitudinal axis to the CG. This torque causes the capsule to rotate in pitch until this torque is balanced by the restoring torques that occur from the symmetrical cone's tilt. At steady state then, the magnitude of the trim angle of attack depends on the CG offset from the longitudinal axis. This value tends to be fairly constant through hypersonic and supersonic flight.

The tilt of the symmetrical cone-shaped capsule at trim creates lift in the direction of the CG offset. As depicted in the figure, the CG offset is constructed to create lift in the general direction of the crew's feet. This upside down posture allows the crew to view the horizon out of the capsule windows.

At speeds above the transonic region, the capsule is stable at trim angle of attack, and aerodynamic torques resist perturbations in angle of attack away from trim. For this reason, pitch and yaw are not directly controlled once in the atmosphere, instead the reaction control thrusters are used to damp pitch and yaw rates to reduce oscillation about trim. In fact, once in the atmosphere, pitch and yaw control torques are insufficient to overcome aerodynamic torques, so controlling to an attitude far from trim is not possible.

The bank angle of the capsule on the other hand is controlled - primarily by the roll thrusters. Control of the bank angle determines the orientation of the lift vector about the relative airflow. The figure depicts zero bank or "lift up." By modulating bank, the direction of lift may be controlled to determine the capsule sink rate. Higher sink rates cause the capsule to encounter denser air more quickly, thereby increasing drag and shortening the downrange flown. In general then, a constant zero bank profile minimizes sink rate and maximizes range, while a constant $180^{\circ}$ bank, or "lift down" profile minimizes range. Guidance may command bank angles between zero and $180^{\circ}$ to achieve the range of the landing site. The resulting heading changes are small, and are compensated for by periodically reversing the bank direction to keep the heading azimuth within preset limits of the landing site bearing.

At zero angle of attack, lift is zero and all aerodynamic force is opposite the air flow in the direction of drag. So the amount of lift generated depends on the CG offset. A particular offset determines the trim angle of attack, which in turn determines the ratio of lift to drag, or L/D ( "L over D"). Larger values of L/D expand the available "footprint" of possible landing sites from a given entry interface condition.

Since $\mathrm{L} / \mathrm{D}$ is determined by the CG offset, the CM designers achieve as much offset as possible by packaging heavy spacecraft components in the desired offset direction. For Orion, this has not resulted in CG offsets which meet $\mathrm{L} / \mathrm{D}$ requirements, so additional ballast must be added on the edge of the capsule cone to create more offset. A design trade must then be performed between

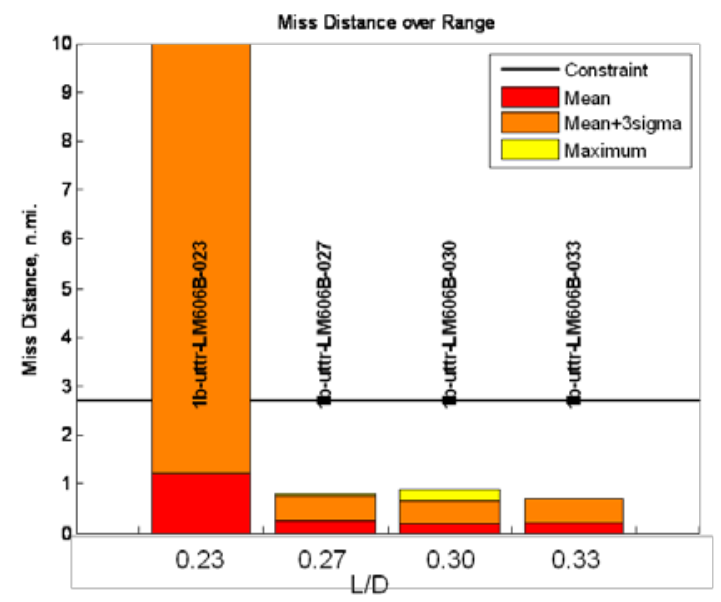

Figure 12. Sensitivity of Miss Distance to $L / D$ the ballast mass, and the performance associated with increased L/D.

To determine the minimum L/D, a series of Monte Carlo analyses were conducted at various entry geometries for both lunar and low Earth orbit (LEO) missions. Figure 12 is an example of the results for lunar return with a constant targeted flight path angle. Note that miss distance is well within the requirement for $\mathrm{L} / \mathrm{D}$ values above 0.23 and the sensitivity of miss remains small with decreasing L/D until suddenly increasing. This "cliff" behavior is due to the fact that the spacecraft can no longer fly the required range to the site due to insufficient lift. 
From these data, the requirement for L/D may be stated as: "The mean lift-to-drag ratio minus three times the standard deviation of the lift-to-drag ratio shall be greater than or equal to 0.23 at Mach 25."

Table 3 contains notional values of the L/D margin that the design ballast mass provides. The sensitivity of $\mathrm{L} / \mathrm{D}$ to ballast mass is a fairly straightforward calculation and is detailed in the appendix?

Table 3. Margin and Sensitivity for $L / D$ versus

Ballast Trade

\begin{tabular}{|l|l|c|c|}
\hline Trade & Requirement/ Design Objective & Margin & Sensitivity (\%/lb) \\
\hline L/D vs Ballast Mass & $\begin{array}{l}\text { Mean L/D }-3 * \sigma \text { of L/D }>=0.23 \\
\text { (lunar) }\end{array}$ & $15 \%$ & 0.05 \\
\hline
\end{tabular}

It should be noted that a maximum $\mathrm{L} / \mathrm{D}$ requirement is also enforced, since high $\mathrm{L} / \mathrm{D}$ values (and their associated trim angles of attack) can cause violations of thermal constraints. However, the minimum L/D value minimizes ballast mass, so it is of most interest for this discussion. For more information about L/D constraints and their associated statistical limitations on CG placement, see reference XXX.

\section{B. Roll Orientation Trades at Touchdown}

As noted above, the Orion crew module is required to have enough habitable volume to carry four astronauts to the Moon with associated life support systems and expendables. The resultant capsule diameter is $16.5 \mathrm{ft}$, nearly $4 \mathrm{ft}$ larger than the Apollo capsule with 2.5 times the internal volume. The larger capsule diameter presents structural design problems, especially during water landings in high sea states. Landing impact attenuation systems designed to protect the crew from, one-chute-out touchdown loads require increased mass and volume to handle worst-case combinations of sink rate, horizontal velocity, and wave impact geometry.

In order to mitigate the risk of structural failure or crew load exceedance the orientation of the capsule is controlled at touchdown to provide the best direction of impact for the attenuation system and to minimize the probability of impacting a wave in a "belly flop" orientation. Figure 13 illustrates the desired orientation with respect to the surface-relative velocity at impact $\left(\mathrm{V}_{\text {rel }}\right)$. Since the horizontal component of the relative velocity is due primarily to wind, changes in wind direction in the final $1500 \mathrm{ft}$ or so prior to touchdown result in changes in relative velocity which must be tracked by the on-board control system.

The ability to respond to changes in horizontal velocity is a function of velocity measurement accuracy and control response time. Accurate measurement of small, winddriven horizontal velocities requires GPS. Even with GPS accuracy, however, the accuracy depends on velocity magnitude, which at steady state equals wind speed. So high winds resulting in large horizontal velocities are easily measured, while low winds make velocity direction determination difficult. Fortunately, roll orientation at low relative speed is not critical for crew loading or structural deformation.

Control response is also a factor in achieving the desired orientation. When wind direction changes within the last few feet of the descent, the control system fires roll thrusters to turn the vehicle toward the new direction. The change in orientation may cause the main chute risers to twist, creating a back-torque disturbance with which the 
controller must contend. Further the controller is required to have a high success rate with the loss of one of the two redundant strings of CM thrusters, so control authority may be reduced in the worst case. Fortunately, in high-wind situations, wind direction variability is reduced and the wind direction is more likely to persist.

Figure 14 is a plot of the touchdown roll error against horizontal velocity magnitude for a Monte Carlo study which modeled wind variations, chute dynamic parameter variations, and navigation errors. The red boundary is the requirement for roll control accuracy, and reflects the maximum allowable value of three times the standard deviation of the error. Note that when the horizontal velocity is less than $10 \mathrm{ft} / \mathrm{s}$, no roll control is required. At speeds greater than $10 \mathrm{ft} / \mathrm{s}$, the allowable error is decreased. This reflects the fact that both crew loads and worstcase structural loads are worse at high horizontal impact speeds. Also note that the shape of the "cloud" of blue error markers reflects the improved navigation accuracy and reduced wind variability at higher wind speeds.

The red requirement boundary in the figure may also be viewed as the system level design "knob" to allow systems engineers to trade the risk of landing in an undesireable orientation against the mass required to make the structure and crew loads attenuation system robust to larger errors at higher gound speeds. Since the shape of the requirement line is complex, a single driving "choke point" is chosen to determine margins and sensitivities. This point is at $40 \mathrm{ft} / \mathrm{s}$ in the figure and the margin is determined by comparing the boundary error at this point $\left( \pm 20^{\circ}\right)$ with three times the standard deviation of the error in the vicinity of the point (for simplicity, we shall assume that the mean error is zero).

Here the mass of interest is the mechanism and structural mass required to limit crew loads and maintain structural integrity. The sensitivity of interest then is the sensitivity of the heading error at $40 \mathrm{ft} / \mathrm{sec}$ to structural and mechanism mass. Notional values of the margin and sensitivity are provided in Table 4.

Table 4. Margin and Sensitivity for Heading Error Trade

\begin{tabular}{|l|c|c|c|}
\hline Trade & Requirement/ Design Objective & Margin & Sensitivity (\%/lb) \\
\hline $\begin{array}{l}\text { Heading error performance vs. } \\
\text { Structural Mass }\end{array}$ & $\begin{array}{l}3^{*} \sigma \text { of heading error }<20 \text { deg at } 40 \\
\mathrm{ft} / \mathrm{sec}\end{array}$ & $15 \%$ & 0.33 \\
\hline
\end{tabular}

\section{Vehicle Design Application and Lessons Learned}

The studies summarized above provided information to the Orion SEIT team and Orion mana gement to make vehicle-level design decisions which are still in process at the time of this writing. This section looks at a process for combining the study results to make design decisions, and provides lessons learned during the conduct of these and other studies. It is important for the analyst to bear in mind the purpose of the analysis since the information must be presented to management in a clear manner, and should include the underlying physics (at a high level), the applicable requirements, the margin on those requirements and the sensitivity of that margin to mass.

\section{A. Margin Balancing}

For the purposes of illustrating some of the elements of a decision making process, we have simplified the study results somewhat so that a classical margin balancing technique may be used to allocate mass between vehicle components. After discussing these process elements, considerations for real world application will be covered.

Table 5 summarizes the data and results of four of the studies presented above. These four studies had attributes that lend themselves well to mathematical margin balancing, namely they relate mass to a required performance or safety metric, and their results provided the margins and sensitivities required to balance margin. In the following discussions, we refer to mass as the "currency" used to balance margin. Since we have a fixed amount of currency (mass) in Orion, the systems engineering decision is how to allocate, or "spend" the currency to optimize performance. 
Table 5. Margin Balancing for Mass Allocation

\begin{tabular}{|l|l|l|c|c|c|c|}
\hline Study & Trade & $\begin{array}{l}\text { Requirement/ } \\
\text { Design Objective }\end{array}$ & Margin & $\begin{array}{l}\text { Sensitivity } \\
(\% / \mathrm{lb})\end{array}$ & $\begin{array}{c}\Delta \mathrm{m} \text { for } \\
\text { Margin } \\
\text { Balance (lb) }\end{array}$ & $\begin{array}{c}\text { Balanced } \\
\text { Margin }\end{array}$ \\
\hline LAS Ballast & $\begin{array}{l}\text { Success vs. } \\
\text { Ballast Mass }\end{array}$ & $\begin{array}{l}80 \% \text { Success at any } \\
\text { altitude }\end{array}$ & $-19 \%$ & 0.23 & +134.9 & $12.0 \%$ \\
\hline $\begin{array}{l}\text { Lunar Surface } \\
\text { Coverage }\end{array}$ & $\begin{array}{l}\text { Surface Coverage } \\
\text { vs. Propellant } \\
\text { Mass }\end{array}$ & $\begin{array}{l}65 \% \text { of lunar surface } \\
\text { acheiveable on } \\
100 \% \text { of launch } \\
\text { epochs }\end{array}$ & $20 \%$ & 0.12 & -66.4 & $12.0 \%$ \\
\hline $\begin{array}{l}\text { Entry } \\
\text { Performance }\end{array}$ & $\begin{array}{l}\text { L/D vs Ballast } \\
\text { Mass }\end{array}$ & $\begin{array}{l}\text { Mean L/D }-3 * \sigma \text { of } \\
\text { L/D }>=0.23 \text { (lunar) }\end{array}$ & $15 \%$ & 0.05 & -59.5 & $12.0 \%$ \\
\hline $\begin{array}{l}\text { Touchdown } \\
\text { Heading } \\
\text { Control }\end{array}$ & $\begin{array}{l}\text { Heading error } \\
\text { performance vs. } \\
\text { Structural Mass }\end{array}$ & $\begin{array}{l}3 * \sigma \text { of heading } \\
\text { error }<20 \text { deg }\end{array}$ & $15 \%$ & 0.33 & -9.0 & $12.0 \%$ \\
\hline
\end{tabular}

Recall that the ballast margin for the LAS is not lifted all the way to orbit as discussed above, so the ballast mass delta shown in the table is multiplied approximately by 8 to arrive at the actual ballast to be housed in the LAS - or around $1000 \mathrm{lb}$.

Once the system level designer has the information of margin and sensitivity to each requirement with respect to a common currency, the margin should be balanced. This may be accomplished by one of these methods:

1. Modify the requirements: It is always a good idea to revisit the relative importance of the various requirements during margin balancing. How important is the abort success rate compared to Lunar surface coverage given abort probabilities and mission objectives? These kinds of decisions must be made at a project level and the role of the Flight Dynamics team and other engineering organizations is to provide accurate, relevant and digestable data to aid in the decision.

2. Re-allocate the currency: A mathematical example of this process is illustrated in the right two columns of the table and the equations which were applied are derieved in the appendix from the straight forward assumptions that the margin must be balanced with zero change in mass, and that the margins are equal on all requirements after balancing.

3. Apply management reserve: The assumption that the mass change must be zero assumes that no mass growth is allowed. As the design matures, management may opt to release mass margin in the form of reduced weight growth allowance or reduced management reserve. This will allow improved performance across the flight regimes in the table, but the margin balancing principals remain the same.

4. A combination of the above: Systems engineers may elect to modify requirements, re-allocate mass and apply reserve.

The Orion systems engineering problem is much more complex that the example presented. There are many more performance metrics, precise sensitivities are often difficult to determine, and mass on the SM is not equivalent to mass on the $\mathrm{CM}$ since they drive different performance metrics. In practice, systems engineers combine classical margin balancing with engineering judgement, but systems engineers should always know their margins, understand sensitivities, and ensure that no margins stand out.

The last of these is the crux of the vehicle-level systems engineering role. The role of the flight dyanamics analyst, on the other hand, is to provide data on margins and sensitivities. The next section discusses some lessons gleaned from these and other flight dyanamics studies which were conducted with the intent of supplying data to make vehicle-level decisions.

\section{B. Use of Time Domain Simulation and Monte Carlo Analysis in Trade Studies}

All of the trade studies described above used time domain simulation to produce the trade results. Time domain simulations techniques have become more and more popular over the last few decades to generate data for vehicle design. Monte Carlo analysis is often used to derive statistical correlations and sensitivities as shown above. This approach has some benefits, including: 
- The process of simulation model development and integration often provides experience and insight to the developer into the governing physics and performance drivers in applicable flight phases (but this may not always be true for the analyst who may not have been involved in model development)

- Modern computer systems are capable of handling hundreds of parameter variations over thousands of simulation runs. These can provide accurate Monte Carlo statistics over the parameter ranges used for the models used

On Orion, Monte Carlo analysis will be used extensively for requirements verification, but it has also been used for requirements development, sensitivity analysis, and vehicle-level design. Design recommendations gleaned from these applications include:

- Understand the underlying physics

- Use Monte Carlo (or other statistical approaches) sparingly in requirements allocation or error budgeting

- Understand when worst-case analysis is conservative and when it is appropriate

- Avoid pitfalls in Monte Carlo analysis for requirements verification

The following paragraphs details these recommendations.

\section{Recommendation: Understand the underlying physics}

The first recommendation is well known, but bears repeating. When new, inexperienced engineers join a project, they are often assigned simulation analysis tasks. These "turn the crank" jobs can allow engineers to begin producing results soon after joining an organization. However, it takes discipline on the part of supervisory personnel to make sure that the simulations are configured correctly, that the statistical attributes of Monte Carlo parameters are properly set, that output statistics are correctly interpreted, and that the analyst develops a knowledge of the underlying physics. We will discuss some of the statistical aspects below, but the system knowledge can only be gained by accompanying the simulation study with theoretical analysis. One rule of thumb: "never be surprised by a simulation result." That is to say that the simulation or Monte Carlo analysis should confirm what the analyst expects or should refine the analysts' estimate. When this is not the case, the analyst must understand why the results do not match expectations. The sim can be wrong!

\section{Recommendation: Use Monte Carlo sparingly in requirements allocation or error budgeting}

Figure 15 is a simple diagram of the Monte Carlo process which will aid in discussing lessons learned. In the process, the statistical properties of the input parameters are defined, then random values are selected for each of the parameters, and a simulation (or other process) is executed using these values. The resulting parameters of interest are recorded, and the process repeated many times. The statistical properties of the output provides information that may be used to decompose requirements, serve as an input for other design processes or to verify existing requirements.

As an example of requirements decomposition, consider the heading error example in the roll orientation trade above. For this example the requirement on the GN\&C substystem was "three times the standard deviation of the

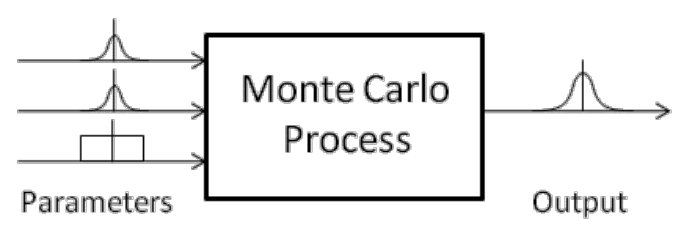

Figure 15. Monte Carlo Process heading error (assuming zero mean) at $40 \mathrm{ft} / \mathrm{sec}$ groundspeed shall be less than $20^{\circ}$." One of the jobs of the Guidance, Navigation and Control (GN\&C) system designer is to decompose this requirement into navigation accuracy and control error allocations. In this example the total error is just the sum of the navigation error and the control error. Here, control error includes error due to disturbances - such as wind gusts wind shear.

One familiar approach to allocating error to navigation and control is the worst case approach. The worst case may be defined as a hard limit, or it may be defined by a project policy such as "design to three sigma." Either way, the total error is the sum of the worst case navigation and control errors. If the total error meets the requirement, then a portion of the margin may be allocated to the lower level requirements in proportion to their contribution. This method is the most conservative method of performing allocation since total error is highly unlikely to exceed the sum of the worst case errors. It is also the simplest method.

If the total error does not meet the requirement, then the systems engineer may elect to petition for a more generous requirement (at a structural mass cost), try to improve navigation and or control performance (perhaps at a 
cost of more expensive hardware or more complex software) - or he may elect to use a less conservative allocation process. One method would be to assume that the total error distribution is a statistical combination of the input distributions. If both navigation and control error are random with Gaussian distribution, then the distribution of the sum of the errors is Gaussian with standard deviation equal to the root-mean-square (RMS) of the inputs.

If the Gaussian statistical assumptions are not applicable to the inputs, or if the relationship between inputs and outputs is complex, then, Monte Carlo analysis is sometimes used to relate the statistical properties of the inputs to the outputs. However, if Monte Carlo is used, two pitfalls must be avoided:

- The output distribution must be close enough to Gaussian to meet the intent of the requirement

- Margin must be applied during requirements allocation

The later pitfall is the most common and causes the most difficulty. If the lower level requirements (navigation and control error specifications in the example) are simply left at their expected " $3 \sigma$ " values, and if there is insufficient margin at the next level (total heading error in the example), then any change in applicable parameters or assumptions may cause an increase in navigation or control variances, which may in turn cause an exceedance of the heading error requirement, which may necessitate redefinition of the requirement, which can affect structural mass and ultimately require reallocation of mass at the vehicle level. In our example, if the landing site changes, and the wind shear statistics worsen, then the control variances will increase, and the effect can propagate up the requirements chain. To prevent this, margin should be consciously allocated early through all requirements levels.

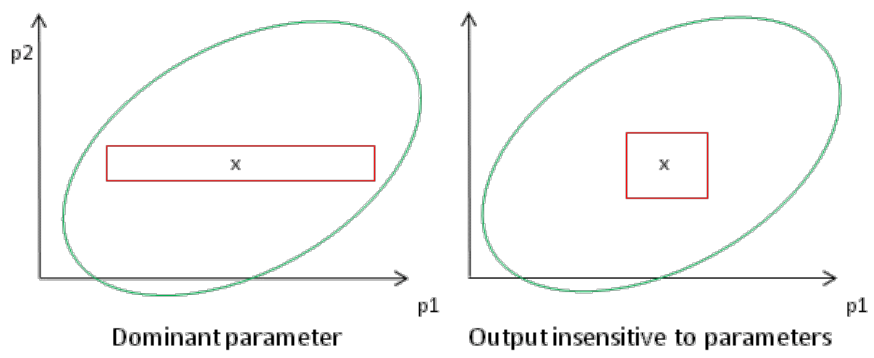

Figure 17. Driving Parameters

\section{Recommendation: Understand when worst-case analysis is conservative and when it is appropriate}

As mentioned above, worst case analysis is a simple approach to requirement decomposition and includes a level of conservatism or "built in" margin. But analysts often confuse the low probabilityof a worst case combination with conservatism. To illustrate this, consider Figure 16 - an abstraction of the input parameter space. The horizontal axis represents the value of an input parameter, "p1" and the vertical axis another parameter, "p2." The mean values of $\mathrm{p} 1$ and $\mathrm{p} 2$ are shown by the cross at center, while the red box represents the range of values between mean minus $3 \sigma$ and mean plus $3 \sigma$. The green ellipse represents the region in the $\mathrm{p} 1, \mathrm{p} 2$ parameter space for which a requirement is met.

The common argument for using a statistical approach to reduce conservatism is to note that the probablitly of the p1, p2 pair existing near the corners of the box is low. [Include equation here. I think it’s (1-99.73)^n]. As the number of parameters increases, the probability of parameters combining at the corners of the box in a "worst on worst" combination rapidly approaches zero. So assessing to the corners of the box that cause requirements exceedance may be unnecessarily conservative.

While it is true that worst case analysis assumes a low probability condition, the degree to which it is conservative depends on the relative sensitivity of the output to the parameters. The left diagram of Figure 17 shows a case where $\mathrm{p} 1$ is a "driving" parameter and the output is relatively insensitive to $\mathrm{p} 2$. In this case, evaluating the corner of the box does not add a significant amount of conservatism - even though the p1, p2 pair at the corner is highly improbable. And the same is true for the right hand diagram, in which the output is not highly sensitive to either parameter in the context of their variances.

It is often the case that a system response is driven largely by one or two parameters, so, depending on the amount of margin available, and the relative sensitivity of the output to the other parameters, a worst case, or "corners of the box" approach to requirement decomposition is often the simplest and most robust method.

This worst case approach was used by the Orion flight dynamics team in defining the requirements for CG offset as discussed in the L/D section above. For missions to the International Space Station, the CG offset was derived by assuming that aerodynamic parameters were at their worst values (when uniformly distributed) or their $3 \sigma$ values when Gaussian. This allowed a simple definition for a CG box with adequate margin for uncertainties and 
operational variations in CG. For the Lunar mission, however, margin was much more constrained - largely by a higher minimum L/D requirement, so a more sophisticated statistical approach was taken, as described in reference XXX.

\section{Recommendation: Avoid pitfalls in Monte Carlo analysis for requirements verification}

As the project approaches CDR, the requirements allocation process is largely complete, and the focus shifts toward proving requirements compliance via pre-CDR verification. During this phase, simulation analysis using higher fidelity models is an appropriate method for assessing requirements compliance. However, several common pitfalls should be avoided:

1. Set parameters according to bounding requirements - not expected values

2. Margin is determined from output results - not input variations

3. ... however, input variations can and should be used to determine robustness - find out where it breaks

4. TBS

\section{Conclusions}

TBS

\section{Appendix}

\section{A. Margin Balancing Equations}

Once margin and sensitivity data are available for each requirement, the equations to re-allocate the independent cost variable (often mass, or monetary cost, or a combination) may be derived as follows:

The change in margin for the $\mathrm{i}^{\text {th }}$ requirement, $\Delta M_{i}$ is added to the existing margin to achieve the balanced margin which we desire to be constant for all requirements:

The change in margin may be replaced by the change in cost variable, $C$ times the sensitivity of margin to the cost variable:

SO

and

Now, when re-allocating cost, the net cost change between all $\mathrm{n}$ requirements must be zero:

$$
=0
$$

SO

$$
=0
$$

This expression contains the constant balanced margin for each requirement, $M_{b}$, and the know values for the margins and sensitivities for each requirement. To solve for $M_{b}$, the common denominator for each of the terms in the sum is multiplied by each sum:

Define:

SO

so the desired balanced margin value is: 
and the changes in cost variable and margin for each requirement are may be determined from 1 , and 2 above.

\section{B. Margin for Statistical Success Requirements}

The approach taken in this exercise was to presume that abort failure is the result of an "underlying performance parameter" that exceeds a limit. We further assume that the underlying parameter has a Gaussian distribution of mean zero and a standard deviation that causes the limit to be exceeded $5 \%$ of the time. This standard deviation then is the required standard deviation of the parameter. We then compute the standard deviation that causes the parameter to exceed the limit at the observed percentage. Margin is then computed as the difference between these standard deviations.

TBS.

Mike Hughes

\section{Acknowledgments}

AAMT leads and analysts

Trajectory leads and analysts (Condon, Williams et. al.)

EMT leads and analysts

\section{References}

The.

Jeremy Rea's paper

Condon's lunar coverage analysis (tech brief?)

Jackson's TEI presentation

Condon, Williams et al TEI tech brief?

LAV Ballast TDS?

Periodicals

${ }^{1}$ Vatistas, G. H., Lin, S., 